\title{
Environmental Chemical Substances in Relation to Neurodevelopmental Disorders: A Systematic Literature Review
}

\author{
Hideko Sone, Tin-Tin Win-Shwe, Xian-Yang Qin, \\ Hiromi Akanuma and Satoshi Imanishi \\ National Institute for Environmental Studies \\ Japan
}

\section{Introduction}

Since the 1950s-70s, increasing number of regulations have rapidly expanded for the global usage of industrial, agricultural and other environmental chemical substances (ECSs). It is believed that children are at high risk of exposure to ECSs, which are produced in quantities greater than one million tons per year and widely dispersed in air, water, food crops, communities, waste sites and homes (Landrigan et al. 2006). Prevalence rates of many common diseases in children, including certain childhood cancers (Devesa et al. 1995; Robison et al. 1995; Schechter 1999; Supriyadi et al. 2011), birth defects (Ananth et al. 2005; Gilboa et al. 2010) and neurodevelopmental disorders (Malik et al. 2011), have been increased or maintained at high levels in industrialized countries. Although primary etiologic factors contributing to these diseases are unknown yet, accumulating evidences indicate that exposure to ECSs are partially responsible for the developmental disabilities, such as autism spectrum disorders (ASDs), attention deficit hyperactivity disorder (ADHD), and other developmental delays (Boyle et al. 2011; Larson et al. 2001) (Figure 1). In this chapter, we conducted a systematic literature review for neurotoxic agents in environment to elucidate the relationship between exposure to ECSs and neurodevelopment disorders in children.

\section{Exposure to ECSs and neurodevelopmental disorders in children}

According to the Fourth National Report on Human Exposure to Environmental Chemicals (Fourth Report), 212 ECSs were detected in the urine and blood samples from the civilian, noninstitutionalized U.S. population (CDC 2009). Therefore, we searched literatures for these 212 ECSs with keywords of "learning disabilities", "developmental delay" and "autism" by PubMed (http://www.ncbi.nlm.nih.gov/pubmed), and then a total of 1075 literatures were selected published during 1972 - 2011 July. These ECSs were categorized by their chemical features in this review (Table 1). Neurodevelopmental disorders in children are disabilities associated primarily with the functionings of the neurological system and brain, which include ADHD, ASDs, mental retardation (MR) and learning disabilities (LD). It has been known that children with neurodevelopmental disorders experience problems 
with language and speech, motor skills, behavior, memory, learning, or other neurological functions. Therefore, we further selected important papers by the review of the keywords, such as children, exposure, neurobehavioral, neurotoxicity, neuropsychological, disabilities, cognitive, development, behavior and school-aged for human study and learning memory, exposure, neurobehavioral, neurodegeneration, neurogenesis, neuropathological, neurotoxicity, impairments, deficits, behavior, hippocampus, developmental, cognitive, disorders, receptors and model for animal studies. Here, a total of 142 literatures for human study (Table 2) and 168 literatures for animal experiments were listed (Table 3).

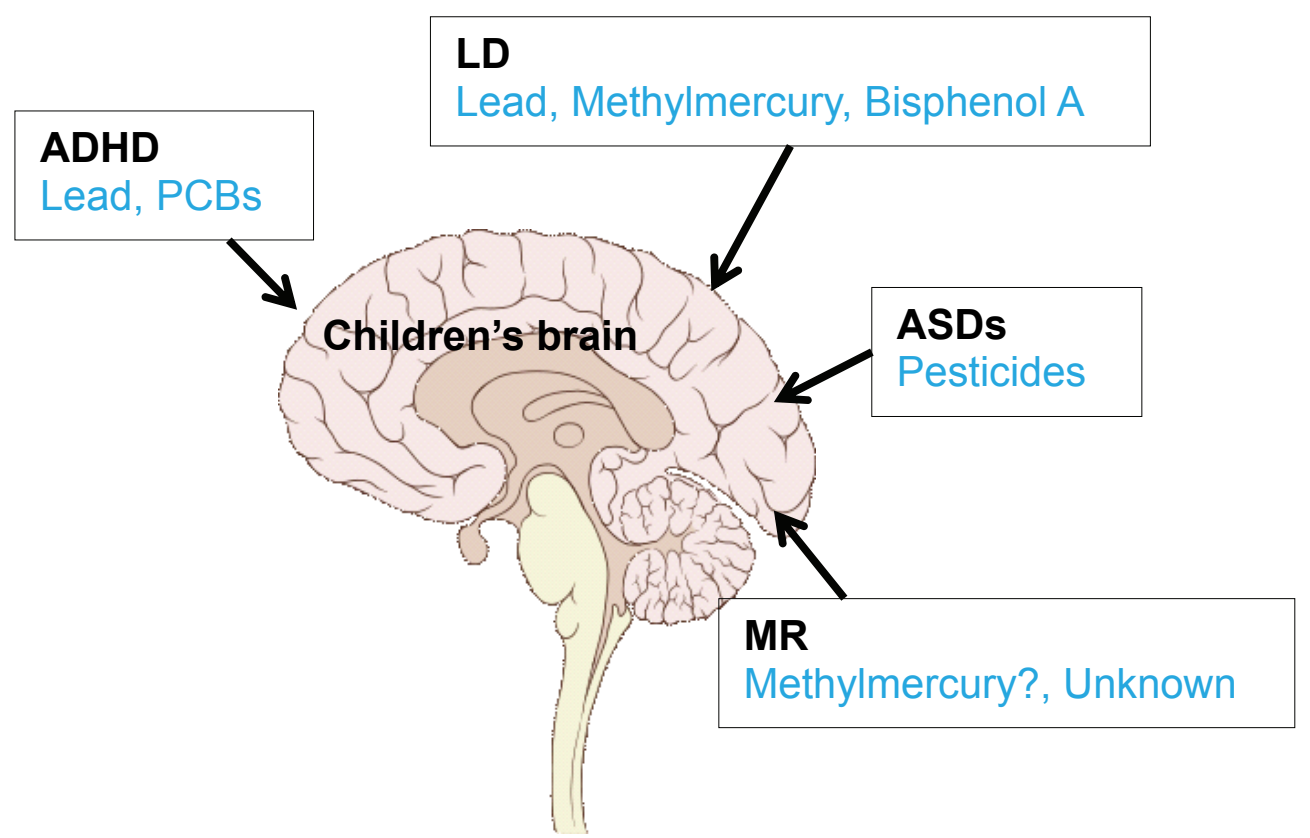

Fig. 1. Relationship between ECSs and neurodevelopmental disorders in children. ADHD: attention deficit hyperactivity disorder, LD: learning disability, ASDs: autism spectrum disorders, MR: mental retardation.

\begin{tabular}{|c|c|}
\hline Categories & Chemical substances \\
\hline Metals & $\begin{array}{l}\text { Antimony, Arsenic, Dimethylarsinic Acid, } \\
\text { Monomethylarsonic Acid, Trimethylarsine oxide, } \\
\text { Barium, Beryllium, Cadmium, Cobalt, Lead, Mercury, } \\
\text { Molybdenum, Platinum, Thallium, Tungsten, Uranium }\end{array}$ \\
\hline $\begin{array}{l}\text { Particulate matters and smoke } \\
\text { Particulate matters }\end{array}$ & $\begin{array}{l}\text { PM10, PM2.5, Carbon monoxide (CO) Sulfur dioxide } \\
\text { (SO2), Nitrogen oxides (NOx), Ozone (O3), Asbestos }\end{array}$ \\
\hline Smoking & $\begin{array}{l}\text { Cotinine, NNAL(4-methylnitrosamino)-1-(3-pyridyl)-1- } \\
\text { butanol) }\end{array}$ \\
\hline
\end{tabular}




\begin{tabular}{|c|c|}
\hline Categories & Chemical substances \\
\hline Agricultural related chemicals & \\
\hline Herbicides and metabolites & $\begin{array}{l}\text { Acetochlor mercapturate, Alachlor mercapturate, } \\
\text { Atrazine mercapturate, 2,4-Dichlorophenoxyacetic acid, } \\
\text { Metolachlor mercapturate, 2,4,5-Trichlorophenoxyacetic } \\
\text { acid, N,N-Diethyl-meta -toluamide (DEET) }\end{array}$ \\
\hline Insecticides and metabolites & Carbamates, Carbofuranphenol, 2-Isopropoxyphenol \\
\hline $\begin{array}{l}\text { Organochlorines and } \\
\text { metabolites }\end{array}$ & $\begin{array}{l}\text { Aldrin, o, } \mathrm{p}^{\prime} \text {-Dichlorodiphenyltrichloroethane, } \mathrm{p}, \mathrm{p}^{\prime} \text { - } \\
\text { Dichlorodiphenyltrichloroethane (DDT), p, p' - } \\
\text { Dichlorodiphenyldichloroethene (DDE), Dieldrin, } \\
\text { Endrin, Hexachlorobenzene, beta - } \\
\text { Hexachlorocyclohexane, gamma- } \\
\text { Hexachlorocyclohexane (Lindane), Heptachlor epoxide, } \\
\text { Mirex, trans -Nonachlor, Oxychlordane, 2,4,5- } \\
\text { Trichlorophenol, 2,4,6-Trichlorophenol }\end{array}$ \\
\hline $\begin{array}{l}\text { Organophosphorus } \\
\text { insecticides: dialkyl phosphate } \\
\text { metabolites }\end{array}$ & $\begin{array}{l}\text { Diethylphosphate (DEP), Dimethylphosphate (DMP), } \\
\text { Diethylthiophosphate (DETP), Dimethylthiophosphate } \\
\text { (DMTP), Diethyldithiophosphate (DEDTP), } \\
\text { Dimethyldithiophosphate (DMDTP) }\end{array}$ \\
\hline $\begin{array}{l}\text { Organophosphorus } \\
\text { insecticides: specific } \\
\text { insecticides and metabolites }\end{array}$ & $\begin{array}{l}\text { 3-Chloro-7-hydroxy-4-methyl-2H-chromen-2-one/ol, 2- } \\
\text { (Diethylamino)-6-methylpyrimidin-4-ol/one, 2- } \\
\text { Isopropyl-4-methyl-6-hydroxypyrimidine, Malathion } \\
\text { dicarboxylic acid, para -Nitrophenol, 3,5,6-Trichloro-2- } \\
\text { pyridinol }\end{array}$ \\
\hline $\begin{array}{l}\text { Pyrethroid pesticide } \\
\text { metabolites }\end{array}$ & $\begin{array}{l}\text { cis -3-(2,2-Dibromovinyl)-2,2-dimethylcyclopropane } \\
\text { carboxylic acid, cis -3-(2,2-Dichlorovinyl)-2,2- } \\
\text { dimethylcyclopropane carboxylic acid, trans -3-(2,2- } \\
\text { Dichlorovinyl)-2,2-dimethylcyclopropane carboxylic } \\
\text { acid, 4-Fluoro-3-phenoxybenzoic acid, 3- } \\
\text { Phenoxybenzoic acid }\end{array}$ \\
\hline \multicolumn{2}{|l|}{ Persistent organic pollutants } \\
\hline Perfluorinated compounds & $\begin{array}{l}\text { Perfluorobutane sulfonic acid (PFBuS), PFDeA, PFDoA, } \\
\text { PFHpA, PFHxS, PFNA, PFOA, PFOS, PFOSA, Et- } \\
\text { PFOSA-AcOH, Me-PFOSA-AcOH, PFUA }\end{array}$ \\
\hline $\begin{array}{l}\text { Polybrominated diphenyl } \\
\text { ethers and polybrominated } \\
\text { biphenyl }\end{array}$ & $\begin{array}{l}\text { 2,2',4-Tribromodiphenyl ether (BDE 17), BDE 28, BDE } 47 \text {, } \\
\text { 2BDE 66, BDE 85, BDE 99, BDE 100, BDE 153, BDE 154, } \\
\text { BDE 183, 2,2',4,4',5,5'-Hexabromobiphenyl (BB 153) }\end{array}$ \\
\hline
\end{tabular}




\begin{tabular}{|c|c|}
\hline Categories & Chemical substances \\
\hline $\begin{array}{l}\text { Polychlorinated biphenyls, } \\
\text { non-dioxin-Like }\end{array}$ & 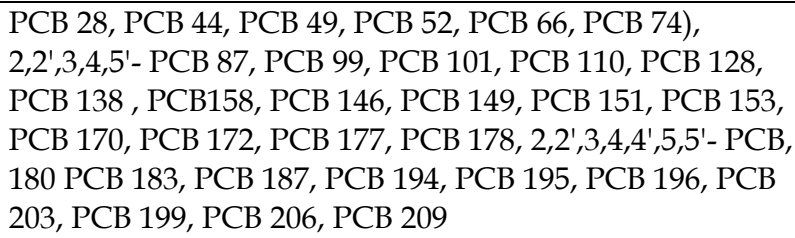 \\
\hline $\begin{array}{l}\text { Dioxin-like polychlorinated } \\
\text { Biphenyls }\end{array}$ & \\
\hline $\begin{array}{l}\text { Coplanar polychlorinated } \\
\text { biphenyls }\end{array}$ & РCB 81, РCB 126, РCB 169 \\
\hline $\begin{array}{l}\text { Mono-ortho-substituted } \\
\text { polychlorinated biphenyls }\end{array}$ & $\begin{array}{l}\text { PCB 105, PCB 118, РCB 156, РCB 157, РСB 167, РCB 189, } \\
\text { 1,2,3,4,6,7,8-Heptachlorodibenzofuran (HpCDF), } \\
1,2,3,4,7,8,9-\end{array}$ \\
\hline Polychlorinated dibenzofurans & $\begin{array}{l}\text { HpCDF, HxCDF, HxCDF, HxCDF, HxCDF, OCDF, } \\
1 \text { PeCDF, PeCDF, TCDF }\end{array}$ \\
\hline $\begin{array}{l}\text { Polychlorinated dibenzo- } p \text { - } \\
\text { dioxins }\end{array}$ & $\begin{array}{l}\text { 2,3,7,8-Tetrachlorodibenzo- } p \text {-dioxin (TCDD), HpCDD, } \\
\text { HxCDD, HxCDD, HxCDD, OCDD, PeCDD, }\end{array}$ \\
\hline $\begin{array}{l}\text { Polycyclic aromatic } \\
\text { hydrocarbon metabolites }\end{array}$ & $\begin{array}{l}\text { 2-Hydroxyfluorene, 3-Hydroxyfluorene, 9- } \\
\text { Hydroxyfluorene, 1-Hydroxynaphthalene (1-Naphthol), } \\
\text { 2-Hydroxynaphthalene (2-Naphthol), 1- } \\
\text { Hydroxyphenanthrene, 2-Hydroxyphenanthrene, 3- } \\
\text { Hydroxyphenanthrene, 4-Hydroxyphenanthrene, 1- } \\
\text { Hydroxypyrene }\end{array}$ \\
\hline $\begin{array}{l}\text { Volatile organic compounds } \\
\text { (VOCs) }\end{array}$ & $\begin{array}{l}\text { Benzene, Chlorobenzene, 1,2-Dibromo-3-chloropropane, } \\
\text { Dibromomethane, 1,2-Dichlorobenzene, m- } \\
\text { Dichlorobenzene, Paradichlorobenzene, 1,1- } \\
\text { Dichloroethane, Ethylene dichloride, Vinylidene } \\
\text { chloride, cis -1,2-Dichloroethene, trans -1,2- } \\
\text { Dichloroethene, Dichloromethane, 1,2-Dichloropropane, } \\
\text { 2,5-Dimethylfuran, Ethylbenzene, Hexachloroethane, } \\
\text { Methyl tert -butyl ether (MTBE), Nitrobenzene, Styrene, } \\
\text { 1,1,2,2-Tetrachloroethane, Perchloroethylene, Carbon } \\
\text { tetrachloride, Toluene, Methyl chloroform, 1,1,2- } \\
\text { Trichloroethane, Trichloroethene Trichloroethylene, o- } \\
\text { Xylene, m- and p -Xylene }\end{array}$ \\
\hline
\end{tabular}




\begin{tabular}{|c|c|}
\hline Categories & Chemical substances \\
\hline \multicolumn{2}{|l|}{ Hormones and environmental } \\
\hline \multicolumn{2}{|l|}{$\begin{array}{l}\text { hormones (endocrine } \\
\end{array}$} \\
\hline \multicolumn{2}{|l|}{ disrupting chemicals) } \\
\hline \multirow[t]{4}{*}{ Environmental phenols } & Bisphenol A (2,2-bis [4-Hydroxyphenyl] propane), \\
\hline & Benzophenone-3 (2-Hydroxy-4-methoxybenzophenone, \\
\hline & 4-tert -Octylphenol (4-[1,1,3,3-Tetramethylbutyl] phenol) \\
\hline & , Triclosan (2,4,4'-Trichloro-2'-hydroxyphenyl ether) \\
\hline Phthalate metabolites & $\begin{array}{l}\text { Mono-benzyl phthalate (MBzP), MiBP, MnBP, MCHP, } \\
\text { MEP, MEHP, MEHHP, MEOHP, MECPP, MiNP, MMP, } \\
\text { MCPP, MOP }\end{array}$ \\
\hline Phytoestrogens and Metabolites & $\begin{array}{l}\text { Daidzein, O-Desmethylangolensin, Enterodiol, } \\
\text { Enterolactone, Equol, Genistein }\end{array}$ \\
\hline \multirow{2}{*}{\multicolumn{2}{|c|}{$\begin{array}{l}\text { Other combustion products and } \\
\text { by-products }\end{array}$}} \\
\hline & \\
\hline Acrylamide Adducts & Acrylamide, glycidamide \\
\hline $\begin{array}{l}\text { Chloromethane related by- } \\
\text { Products }\end{array}$ & $\begin{array}{l}\text { Bromodichloromethane, dibromochloromethane } \\
\text { (chlorodibromomethane), Tribromomethane } \\
\text { (bromoform), Trichloromethane (chloroform) }\end{array}$ \\
\hline
\end{tabular}

Table 1. List of ECSs detected in human blood and urine that has been related with LD.

\subsection{Association between ECSs exposure with LD}

Studies have found that several widespread environmental contaminants can damage the children's developing brains and nervous systems. In our literature review, lead, methylmercury, pesticides, tobacco (cotinine), persistent organic pollutants such as polychlorinated biphenyls (PCBs), and environmental hormones such as bisphenol A and phthalates have been indicated association between neuronal disability and exposure levels in children (Table 3). For instances, prenatal tobacco and childhood lead exposures may be significant risk factors for ADHD, especially when individuals are exposed to both of these toxicants (Froehlich et al. 2009). Although the U.S. has made considerable strides in reducing these toxicant exposure, $15 \%$ of women reported smoking during pregnancy in the U.S. population-based study in 2004 (Allen et al. 2008), and an estimated 1.6\% of U.S. children showed blood lead levels of concern $(\geq 10 \mu \mathrm{g} / \mathrm{dL})$ in 1999-2002, with almost $14 \%$ having levels of 5 to $9 \mu \mathrm{g} / \mathrm{dL}$ (CDC 2005). These findings suggest that reduction of toxicant exposure may be an important role for the preventions of ADHD as well as other neurodevelopmental disorders in children.

In addition, the accumulating evidences suggest a link between lead exposure and memory impairment. van Wijngaarden et al. (2009) conducted a pilot study of 47 healthy subjects aged 55-67 years to examine associations between bone lead levels and 4 tests sensitive to the natural history of Mild Cognitive Impairment (MCI) and Alzheimer's disease (AD), which included 3 subtests of the Cambridge Neuropsychological Test Automated Battery (delayed match-to-sample, paired associates learning and spatial recognition memory) and 
the Montreal Cognitive Assessment Test. By measurements of bone lead concentrations, higher tibial and calcaneal bone lead values were significantly $(p<0.05)$ associated with lower performance levels on delayed match-to-sample and paired associates learning in unadjusted analyses with Spearman rank correlation coefficients of about 0.4. Multiple linear regression analyses (i.e., least-squares means of cognitive test scores across tertiles of lead exposure) adjusted for age, education and smoking status continued to show an association of higher calcaneal lead levels with increasing memory impairments on delayed match-to-sample $(\mathrm{p}=0.07)$. As might be expected, additional adjustment for history of hypertension reduced the strength of this association $(p=0.19)$. Given the demonstrated impact of lead exposure on hypertension and the vascular aetiology of certain dementias, authors speculated that hypertension could play a mediating role in the association between lead exposure and memory impairment.

Pesticides and their degradation products are ubiquitous in the environment. The most commonly detected indoor pesticides (organophosphates and pyrethroids), which are routinely applied in classrooms and playgrounds, are well-known neurotoxicants that affect the ability to learn and process information (Tulve et al. 2006). In our literature survey, $\mathrm{Xu}$ et al. (2011) examined the association between body burden of trichlorophenol (TCP) (ie, 2,4,5TCP and 2,4,6-TCP) and ADHD by logistic regression analyses using data from the 19992004 National Health and Nutrition Examination Survey (NHANES) to evaluate the association between urinary TCPs and parent-reported ADHD among 2546 children aged 615 years. Their results showed that children with low levels $(<3.58 \mu \mathrm{g} / \mathrm{g})$ and high levels $(\geq 3.58 \mu \mathrm{g} / \mathrm{g}$ ) of urinary 2,4,6-TCP had a higher risk of parent-reported ADHD compared to children with levels below the limit of detection (OR 1.54, 95\% CI 0.97 to 2.43 and OR 1.77, $95 \%$ CI 1.18 to 2.66 , respectively; $p$ for trend $=0.006$ ) after adjusting for covariates. No association was found between urinary 2,4,5-TCP and parent-reported ADHD. These results suggested that exposure to TCP may increase the risk of behavioural impairment in children, especially in countries where organochlorine pesticides are still commonly used. It also should be noted that Rauh et al. (2006) reported the impact of prenatal exposure to chlorpyrifos on 3-year neurodevelopment and behavior in city-residential minority 254 children. The report examined cognitive and motor development with the Bayley Scales of Infant Development II and child behavior with the Child Behavior Checklist and chlorpyrifos levels in umbilical cord plasma. Highly exposed children (chlorpyrifos levels of $>6.17 \mathrm{pg} / \mathrm{g}$ plasma) scored, on average, 6.5 points lower on the Bayley Psychomotor Development Index and 3.3 points lower on the Bayley Mental Development Index at 3 years of age compared with those with lower levels of exposure. Children exposed to higher in compared with lower chlorpyrifos levels were also significantly more likely to experience Psychomotor Development Index and Mental Development Index delays, which are attention problems, ADHD problems, and pervasive developmental disorder problems at 3 years of age. The proportion of delayed children in the high-exposure group, compared with the low-exposure group, was 5 times greater for the Psychomotor Development Index and 2.4 times greater for the Mental Development Index.

It was also reported that children prenatally exposed to PCBs might be related with lowered intelligence and behavioral deficits. Relationships between adverse health effects and PCB exposure during infancy and childhood have been examined. Although some inconsistencies in the literature exist, the overall evidence supports a concern for adverse 
effects of PCBs on children's neurological development. It should be noted that adverse effects on intelligence and behavior have been found in girls who were highly exposed to mixtures of PCBs, chlorinated dibenzofurans, and other pollutants prior to conception (Chen et al. 1992; Chen et al. 1994).

\begin{tabular}{|c|c|c|}
\hline Chemicals & Study type/hazard effects & References \\
\hline Arsenic & $\begin{array}{l}\text { Encephalopathy: an } \\
\text { uncommon manifestation of } \\
\text { workplace arsenic poisoning? }\end{array}$ & Morton and Caron 1989 \\
\hline Arsenic & $\begin{array}{l}\text { Neuropsychological } \\
\text { impairment following } \\
\text { inorganic arsenic exposure }\end{array}$ & Bolla-Wilson and Bleecker 1987 \\
\hline Cobalt & $\begin{array}{l}\text { Hair element content in } \\
\text { learning disabled children }\end{array}$ & Pihl and Parkes 1977 \\
\hline Cobalt & $\begin{array}{l}\text { Evidence for interactions of } \\
\text { lithium with vitamin B12 and } \\
\text { with other trace elements }\end{array}$ & Schrauzer et al. 1992 \\
\hline Copper & Dose-effect relationships & Bowler et al. 2007 \\
\hline Copper & $\begin{array}{l}\text { Hair mineral analysis and } \\
\text { behavior: an analysis of } 51 \\
\text { studies }\end{array}$ & Rimland and Larson 1983 \\
\hline Copper & $\begin{array}{l}\text { Manganese inhibits NMDA } \\
\text { receptor channel function: } \\
\text { implications }\end{array}$ & Guilarte and Chen 2007 \\
\hline Copper & $\begin{array}{l}\text { Poor cognitive development } \\
\text { and abdominal pain: Wilson's } \\
\text { disease }\end{array}$ & Gronlund et al. 2006 \\
\hline Copper, Zinc & Brain and behavior & Pfeiffer and Braverman 1982 \\
\hline $\begin{array}{l}\text { Copper, } \\
\text { mercury }\end{array}$ & School children & Capel et al. 1981 \\
\hline Lead, arsenic & Two metals, ADHD & Calderon et al. 2001 \\
\hline Lead & The Edinburgh Lead Study & Thomson et al. 1989 \\
\hline Lead & $\begin{array}{l}\text { Cognition in children and } \\
\text { very low lead exposure }\end{array}$ & Minder et al. 1998; Surkan et al. 2007 \\
\hline Lead, smoke & verbal memory, ADHD & Bleecker et al. 2005; Braun et al. 2006 \\
\hline Lead & Preschool children & $\begin{array}{l}\text { Bellinger et al. 1987; Bellinger et al. 1991; } \\
\text { Benetou-Marantidou et al. 1988; } \\
\text { Fergusson et al. 1997; Jusko et al. 2008; } \\
\text { McMichael et al. 1988; Mendelsohn et al. } \\
\text { 1998; Rabinowitz et al. 1992; Stokes et al. } \\
\text { 1998; (Schwartz et al. 2000; Ris et al. 2004; } \\
\text { Shih et al. 2006; Schwartz et al. 2007; van } \\
\text { Wijngaarden et al. 2009; Mahmoudian et } \\
\text { al. } 2009\end{array}$ \\
\hline
\end{tabular}




\begin{tabular}{|c|c|c|}
\hline Chemicals & Study type/hazard effects & References \\
\hline Lead & $\begin{array}{l}\text { School children } \\
\text { (ADHD, learning disabilities) }\end{array}$ & $\begin{array}{l}\text { Fergusson and Horwood 1993; Lyngbye } \\
\text { et al. 1990; Tong et al. 1996; Tuthill 1996; } \\
\text { Leviton et al. 1993; Minder et al. 1994; } \\
\text { Buchanan et al. 1999; al-Saleh et al. 2001; } \\
\text { Lanphear et al. 2005; McMichael et al. } \\
\text { 1988; Canfield et al. 2003; Chiodo et al. } \\
\text { 2007; Counter et al. 2008; Nigg et al. 2008; } \\
\text { Schnaas et al. 2006; Wang et al. 2002; } \\
\text { Wang et al. 2008; Kim et al. } 2010\end{array}$ \\
\hline $\begin{array}{l}\text { Lead, mercury, } \\
\text { PCB }\end{array}$ & $\begin{array}{l}\text { Children study three } \\
\text { chemicals }\end{array}$ & Stewart et al. 2006 \\
\hline Mercury & infant immunizations & Redwood et al. 2001 \\
\hline Mercury & Children study & $\begin{array}{l}\text { Grandjean et al. 1997; Schettler 2001; } \\
\text { Counter et al. 2005; Johansson et al. 2007; } \\
\text { Dufault et al. 2009; Valent et al. } 2011\end{array}$ \\
\hline Mercury & Adult exposure & Yokoo et al. 2003 \\
\hline Mercury & Workers & $\begin{array}{l}\text { Piikivi and Hanninen 1989; Counter et al. } \\
2005\end{array}$ \\
\hline Molybdenum & A case report & Momcilovic 1999 \\
\hline $\begin{array}{l}\text { Phosphorus-31 } \\
\text { magnetic }\end{array}$ & Children study & Moss et al. 1997 \\
\hline $\begin{array}{l}\text { Carbon } \\
\text { monoxide }\end{array}$ & Children study & Binder and Roberts 1980 \\
\hline $\begin{array}{l}\text { Carbon } \\
\text { monoxide }\end{array}$ & Workers & Deschamps et al. 2003; Katirci et al. 2011 \\
\hline Cotinine & Adult Patients study & Smith et al. 2009 \\
\hline Smoking & Children study & $\begin{array}{l}\text { Marshall et al. 1995; Robertson and } \\
\text { Jackson } 1996 \text { Lassen and Oei 1998; } \\
\text { Rowland et al. 2002; Batstra et al. 2003; } \\
\text { Najman et al. 2004; O'Brien et al. 2004; } \\
\text { Matsumoto et al. 2005; Uzun and Kendirli } \\
\text { 2005b; Kukla et al. 2008; Petry et al. 2008; } \\
\text { Kargoshaie et al. 2009; Anderko et al. } \\
\text { 2010; DeGarmo et al. 2010; Freire et al. } \\
\text { 2010; O'Callaghan et al. 2010 }\end{array}$ \\
\hline Smoking & Adolescent student study & $\begin{array}{l}\text { Ivanovic et al. 2000; Molina and Pelham } \\
\text { 2001; Kalyva 2007; Keselyak et al. 2007; } \\
\text { Wang et al. 2009b }\end{array}$ \\
\hline Pesticide & A case study. & Reidy et al. 1994 \\
\hline Pesticide & Children study & $\begin{array}{l}\text { Stephens et al. 1995; Schettler 2001; } \\
\text { Kofman et al. 2006; Rauh et al. 2006; Xu et } \\
\text { al. } 2011\end{array}$ \\
\hline Pesticide & Workers & Srivastava et al. 2000 \\
\hline
\end{tabular}




\begin{tabular}{|c|c|c|}
\hline Chemicals & Study type/hazard effects & References \\
\hline DDT & Children study & $\begin{array}{l}\text { Christenson et al. 2001; Griffin et al. 1993; } \\
\text { Aase et al. 2006; Christenson et al. 1991; } \\
\text { Dorner and Plagemann 2002; Sharma et } \\
\text { al. } 2009\end{array}$ \\
\hline Dioxin & Children study & van den Hazel et al. 2006; Lee et al. 2007 \\
\hline Formaldehyde & Children study & Madrid et al. 2008 \\
\hline Formaldehyde & Workers & LoSasso et al. 2001 \\
\hline PCBs & Children study & $\begin{array}{l}\text { Chen et al. 1992; Chen et al. 1994; Roegge } \\
\text { and Schantz 2006; Lin et al. } 2010\end{array}$ \\
\hline $\begin{array}{l}\text { Polyaromatic } \\
\text { hydrocarbon }\end{array}$ & Children study & Sheng et al. 2010 \\
\hline $\begin{array}{l}\text { Polychlorinated } \\
\text { biphenyl }\end{array}$ & Children study & Sandberg et al. 2003; Lin et al. 2008 \\
\hline $\begin{array}{l}\text { Polycyclic } \\
\text { aromatic } \\
\text { hydrocarbon }\end{array}$ & Children study & $\begin{array}{l}\text { Walhovd et al. 2007; Bandstra et al. 2010; } \\
\text { van Elderen et al. } 2010\end{array}$ \\
\hline Phenol & Children study & $\begin{array}{l}\text { Gross et al. 1987; Hertz-Picciotto et al. } \\
2011\end{array}$ \\
\hline $\begin{array}{l}\text { Industrial } \\
\text { solvents }\end{array}$ & Children study & Uzun and Kendirli 2005a; Dick et al. 2010 \\
\hline $\begin{array}{l}\text { Industrial } \\
\text { solvents }\end{array}$ & Workers & $\begin{array}{l}\text { Hanninen et al. 1976; Ryan et al. 1988; } \\
\text { Moen et al. 1990; Morrow et al. 1992; } \\
\text { Stollery and Flindt 1988; Bowler et al. } \\
\text { 2001; LoSasso et al. 2001; Morrow et al. } \\
\text { 2001; LoSasso et al. } 2002\end{array}$ \\
\hline
\end{tabular}

Table 2. Literature lists for the hazard effects of ECSs on human memory and cognition.

\subsection{Possible mechanism of ECSs-induced autism and developmental delay}

As above mentioned, several ECSs have been related with developmental delay such as ADHD and LD in human studies. However, little is known about the underlying mechanism by which ECSs could induce developmental delay. Animal experiments and in vitro studies using cells are useful to elucidate these kinds of mechanisms and understand the results of human studies. Animal studies listed in Table 3 indicate that most attentions have been focused on lead, mercury, pesticides and polycyclic aromatic hydrocarbons (PAHs). For example, toxic properties of lead have been attributed to its capability to mimic calcium and alter calcium homeostasis. One of the reasons for the deleterious effects of lead is its ability to strongly bind to sulfhydryl groups of proteins and to mimic or compete with calcium (Flora et al. 2007). It is known that lead, even at picomolar concentration, competes with calcium for binding sites on cerebellar phosphokinase $\mathrm{C}$, thereby affecting neuronal signaling and neurotransmitter release (Bressler and Goldstein 1991), inhibiting calcium entry into cells (Simons 1993). Lead disrupts mitochondrial calcium homeostasis, intercellular oxidants levels, ATP production, and apoptogenic factors. 
Rats in the benzo(a)pyrene $(\mathrm{B}(\mathrm{a}) \mathrm{P})$-treated groups have significantly impaired Morris water maze performance when compared to controls (Chengzhi et al. 2011). The B(a)P-induced neuronal damage was found in the hippocampus under transmission electron microscopy. Their results demonstrated that LM deficits associated protein expression signatures could be identified from tissue proteomes, as well as potential biomarkers such as retinoic acid receptor $b(\mathrm{RARb})$, synaptotagmin iosfomrs 1 (Syt1) and brain-derived neurotrophic factor (BDNF). This finding is the first time that multiple novel proteins that are dysregulated by $\mathrm{B}(\mathrm{a}) \mathrm{P}$, which both enhance our understanding of $\mathrm{B}(\mathrm{a}) \mathrm{P}$ induced locomotor deficits and represent targets of novel therapeutics. Prenatal morphine can alter the synaptic complex of postsynaptic density 95 with N-methyl-D-aspartate receptor subunit in hippocampal CA1 subregion of rat offspring leading to long-term cognitive deficits (Lin et al. 2009). This morphine model might be useful for understanding mechanisms of long-term cognitive deficit induced by other ECSs such as lead and PCBs.

Originally, organophosphate pesticides (OPs) have been thought to exert their effects on brain development secondarily by their ability to inhibit cholinesterase. However, it became now clear that these agents act as developmental neurotoxicants through a number of differential mechanisms. Some of which operate at exposures below the threshold for cholinesterase inhibition may differ in their effects on brain development and their consequent impacts on behavioral performance (Paul et al. 1994; Cohn and MacPhail 1997; Itoh et al. 1997a, 1997b; Palumbo et al. 2001; Castillo et al. 2002; Levin et al. 2002; Aldridge et al. 2005; Spowart-Manning and van der Staay 2005; Timofeeva, 2008; Verma et al. 2009; Levin et al. 2010). A series of studies with toxico-dynamically equivalent exposures in neonatal rats showed that chlorpyrifos, diazinon and parathion (PRT) elicit behavioral abnormalities in association with adverse effects on acetylcholine (ACh) and serotonin (5HT) circuits, but that the underlying defects and behavioral outcomes differ among the three OPs. In particular, PRT exposure did not elicit the cognitive impairment noted with the other two OPs, as evaluated in the radial-arm maze in adolescence and young adulthood, although, it did share adverse effects on indices of ACh synaptic function.

\begin{tabular}{|l|l|l|l|}
\hline \multicolumn{1}{|c|}{ Chemicals } & $\begin{array}{l}\text { Animal } \\
\text { Species }\end{array}$ & \multicolumn{1}{|c|}{$\begin{array}{c}\text { Models and experimental } \\
\text { types }\end{array}$} & \multicolumn{1}{c|}{ References } \\
\hline Arsenic & mice & Behavioral analysis & Miyagawa et al. 2007 \\
\hline Arsenic & mice & Mechanistic study & Martinez-Finley et al. 2009 \\
\hline Arsenic & rats & $\begin{array}{l}\text { Developmental exposure } \\
\text { and behavioral analysis }\end{array}$ & Rodriguez et al. 2002 \\
\hline Arsenic & rats & Mechanistic study & Rodriguez et al. 2001 \\
\hline $\begin{array}{l}\text { Aluminium- } \\
\text { maltolate } \\
\text { complex }\end{array}$ & mice & Mechanistic study & Kaneko et al. 2006 \\
\hline Aluminum & rats & Mechanistic study & Sethi et al. 2009 \\
\hline Aluminum & rats & $\begin{array}{l}\text { Aluminum-induced memory } \\
\text { deficit model rats }\end{array}$ & Gong et al. 2006 \\
\hline Copper & mice & $\begin{array}{l}\text { Behavioral analysis in } \\
\text { Alzheimer's disease models }\end{array}$ & Grossi et al. 2009 \\
\hline
\end{tabular}




\begin{tabular}{|c|c|c|c|}
\hline Chemicals & $\begin{array}{l}\text { Animal } \\
\text { Species }\end{array}$ & $\begin{array}{c}\text { Models and experimental } \\
\text { types }\end{array}$ & References \\
\hline Copper & mice & $\begin{array}{l}\text { Indicator in the Alzheimer's } \\
\text { disease model }\end{array}$ & Fisher et al. 1991; Quinn et al. 2010 \\
\hline Copper & mice & Mechanistic study & Lu et al. 2006; Lu et al. 2009 \\
\hline Copper & hamster & Mechanistic study & Bareggi et al. 2009 \\
\hline Copper & rats & Mechanistic study & $\begin{array}{l}\text { Obernier et al. 2002; Begum et al. } \\
2008\end{array}$ \\
\hline Copper & rats & Competition with zinc & Railey et al. 2010 \\
\hline Copper & rabbit & Mechanistic study & Sparks and Schreurs 2003 \\
\hline Copper & rabbit & $\begin{array}{l}\text { Indicator in the Alzheimer's } \\
\text { disease model }\end{array}$ & Woodruff-Pak et al. 2007 \\
\hline Cobalt & rats & Mechanistic study & Nerobkova and Voronina 1988 \\
\hline Lead & mice & Mechanistic study & Gao et al. 2005; Railey et al. 2011 \\
\hline Lead & mice & $\begin{array}{l}\text { Developmental exposure } \\
\text { and behavioral analysis }\end{array}$ & Garavan et al. 2000 \\
\hline Lead & rats & Mechanistic study & $\begin{array}{l}\text { Alkondon et al. 1990; Adhami et al. } \\
2000 \text { Zhang et al. 2002; Garcia- } \\
\text { Arenas et al. 2004; Vazquez and } \\
\text { Pena de Ortiz 2004; Haider et al. } \\
\text { 2005; Flora et al. 2007 }\end{array}$ \\
\hline Lead & rats & $\begin{array}{l}\text { Developmental exposure } \\
\text { and behavioral analysis }\end{array}$ & $\begin{array}{l}\text { Kumar and Desiraju 1992; Altmann } \\
\text { et al. 1993; Yang et al. } 2003\end{array}$ \\
\hline Lead & rats & Behavioral analysis & Tang et al. 1994; Fan et al. 2009 \\
\hline Mercury & mice & $\begin{array}{l}\text { Prenatal exposure and } \\
\text { Behavioral analysis }\end{array}$ & Montgomery et al. 2008 \\
\hline Mercury & mice & $\begin{array}{l}\text { Developmental exposure } \\
\text { and behavioral analysis }\end{array}$ & $\begin{array}{l}\text { Yoshida et al. 2005; Eddins et al. } \\
2008\end{array}$ \\
\hline Mercury & rats & Mechanistic study & Vicente et al. 2004 \\
\hline Mercury & rats & $\begin{array}{l}\text { Developmental exposure } \\
\text { and behavioral analysis }\end{array}$ & $\begin{array}{l}\text { Sakamoto et al. 2002; Falluel-Morel } \\
\text { et al. } 2007\end{array}$ \\
\hline Mercury & rats & $\begin{array}{l}\text { Dose-dependent study of } \\
\text { Developmental exposure }\end{array}$ & Sakamoto et al. 2004 \\
\hline Mercury & monkey & $\begin{array}{l}\text { Developmental exposure } \\
\text { and behavioral analysis }\end{array}$ & Hellberg 1972 \\
\hline Uranium & rats & Behavioral analysis & $\begin{array}{l}\text { Albina et al. 2005; Houpert et al. } \\
2007\end{array}$ \\
\hline Uranium & rats & $\begin{array}{l}\text { Developmental exposure } \\
\text { and behavioral analysis }\end{array}$ & Sanchez et al. 2006 \\
\hline Vanadium & mice & Mechanistic study & Han et al. 2008 \\
\hline Vanadium & mice & Behavioral analysis & Avila-Costa et al. 2006 \\
\hline Vanadium & rats & Mechanistic study & Mao et al. 2008 \\
\hline $\begin{array}{l}\text { Carbon } \\
\text { monoxide }\end{array}$ & mice & Behavioral analysis & Meunier et al. 2006 \\
\hline
\end{tabular}




\begin{tabular}{|c|c|c|c|}
\hline Chemicals & $\begin{array}{l}\text { Animal } \\
\text { Species }\end{array}$ & $\begin{array}{l}\text { Models and experimental } \\
\text { types }\end{array}$ & References \\
\hline $\begin{array}{l}\text { Carbon } \\
\text { monoxide }\end{array}$ & \begin{tabular}{|l|} 
rats \\
\end{tabular} & Mechanistic study & Thom et al. 2004 \\
\hline $\begin{array}{l}\text { Carbon } \\
\text { monoxide }\end{array}$ & rats & $\begin{array}{l}\text { Mechanistic study and } \\
\text { Behavioral analysis }\end{array}$ & Han et al. 2007; Wang et al. 2009a \\
\hline $\begin{array}{l}\text { Nitrogen } \\
\text { oxide }\end{array}$ & mice & $\begin{array}{l}\text { Mechanistic study and } \\
\text { Behavioral analysis }\end{array}$ & $\begin{array}{l}\text { Reddy and Kulkarni 1998; Palumbo } \\
\text { et al. } 2007\end{array}$ \\
\hline $\begin{array}{l}\text { Nitrogen } \\
\text { oxide }\end{array}$ & rats & $\begin{array}{l}\text { Mechanistic study and } \\
\text { Behavioral analysis }\end{array}$ & $\begin{array}{l}\text { Jevtovic-Todorovic et al. 2003; } \\
\text { Kumar and Kumar 2009; Comin et } \\
\text { al. } 2010\end{array}$ \\
\hline $\begin{array}{l}\text { Nitrogen } \\
\text { oxide }\end{array}$ & rats & Behavioral analysis & Paul et al. 2003 \\
\hline $\begin{array}{l}\text { NNAL(4- } \\
\text { methylnitrosa } \\
\text { mino)-1-(3- } \\
\text { pyridyl)-1- } \\
\text { butanol) }\end{array}$ & hamster & $\begin{array}{l}\text { Use of precision-cut tissue } \mathrm{s} \\
\text { Behavioral analysis }\end{array}$ & Richter et al. 2000 \\
\hline Ozone & rats & $\begin{array}{l}\text { Mechanistic study and } \\
\text { Behavioral analysis }\end{array}$ & Guerrero et al. 1999 \\
\hline Smoking & mice & Behavioral analysis & Paz et al. 2007 \\
\hline Smoking & rats & Mechanistic study & Liang et al. 2006 \\
\hline Smoking & rats & $\begin{array}{l}\text { Developmental exposure } \\
\text { and behavioral analysis }\end{array}$ & Levin et al. 1996 \\
\hline Pesticide & mice & Gufosinate-ammonium & Calas et al. 2008 \\
\hline Pesticide & mice & Organophosphates & $\begin{array}{l}\text { Billauer-Haimovitch et al. 2009; Post } \\
\text { et al. } 2011\end{array}$ \\
\hline Pesticide & mice & $\begin{array}{l}\text { quaternary ammonium } \\
\text { herbicide }\end{array}$ & Chen et al. 2010 \\
\hline Pesticide & rats & Aconitine Mechanistic study & Curzon et al. 2006 \\
\hline Pyrethroid & rats & $\begin{array}{l}\text { Pyrethroid behavioral } \\
\text { analysis }\end{array}$ & Sinha et al. 2006 \\
\hline Pesticide & rats & Organophosphates & $\begin{array}{l}\text { Cohn and MacPhail 1997; Paul et al. } \\
1994 \text { Itoh et al. 1997a; Itoh et al. } \\
\text { 1997b; Palumbo et al. 2001; Castillo } \\
\text { et al. 2002; Levin et al. 2002; } \\
\text { Aldridge et al. 2005; Spowart- } \\
\text { Manning and van der Staay 2005; } \\
\text { Timofeeva, 2008; Verma et al. 2009; } \\
\text { Levin et al. } 2010\end{array}$ \\
\hline Pesticide & rats & Clozapine & Levin et al. 2009 \\
\hline Pesticide & rats & Vinclozolin fungicide & Andre, 2006 \\
\hline Pesticide & rats & DEET & Abdel-Rahman, 2004 \\
\hline Dioxin & mice & Mechanistic study & Akahoshi, 2009 \\
\hline
\end{tabular}




\begin{tabular}{|c|c|c|c|}
\hline Chemicals & $\begin{array}{l}\text { Animal } \\
\text { Species }\end{array}$ & $\begin{array}{l}\text { Models and experimental } \\
\text { types }\end{array}$ & References \\
\hline Dioxin & rats & Mechanistic study & Marcus, 2005 \\
\hline Formaldehyde & mice & Mechanistic study & Tong et al. 2011 \\
\hline Formaldehyde & rats & Mechanistic study & Aslan, 2006; Liu, 2010 \\
\hline $\begin{array}{l}\text { Hexachloro- } \\
\text { Benzene }\end{array}$ & rats & Behavioral analysis & Valkusz, 2011 \\
\hline PCB & rats & $\begin{array}{l}\text { Developmental exposure } \\
\text { and behavioral analysis }\end{array}$ & $\begin{array}{l}\text { Piedrafita, 2008; Boix, } 2010 \text {; Jolous- } \\
\text { Jamshidi et al. } 2010\end{array}$ \\
\hline PCB & fishes & Behavioral analysis & Schantz, 2001 \\
\hline PFOS & mice & $\begin{array}{l}\text { Developmental exposure } \\
\text { and behavioral analysis }\end{array}$ & Johansson et al. 2008 \\
\hline $\begin{array}{l}\text { Polycyclic } \\
\text { aromatic } \\
\text { hydrocarbon }\end{array}$ & mice & ADHD model & Fredriksson and Archer 2004 \\
\hline $\begin{array}{l}\text { Polycyclic } \\
\text { aromatic } \\
\text { hydrocarbon }\end{array}$ & mice & $\begin{array}{l}\text { Behavioral analysis Down } \\
\text { syndrome model }\end{array}$ & Rueda et al. 2008 \\
\hline $\begin{array}{l}\text { Polycyclic } \\
\text { aromatic } \\
\text { hydrocarbon }\end{array}$ & rats & ADHD model & $\begin{array}{l}\text { Chengzhi et al. 2011; Lin et al. 2009; } \\
\text { Nishio et al. } 2001\end{array}$ \\
\hline $\begin{array}{l}\text { Polycyclic } \\
\text { aromatic } \\
\text { hydrocarbon }\end{array}$ & rats & Behavioral analysis & $\begin{array}{l}\text { Sun, 2005; Fedotova and Ordyan } \\
2010\end{array}$ \\
\hline $\begin{array}{l}\text { Polycyclic } \\
\text { aromatic } \\
\text { hydrocarbon }\end{array}$ & rats & $\begin{array}{l}\text { Developmental exposure } \\
\text { and behavioral analysis }\end{array}$ & Benetti, 2009 \\
\hline $\begin{array}{l}\text { Polycyclic } \\
\text { aromatic } \\
\text { hydrocarbon }\end{array}$ & rats & Adult behavioral analysis & Revest, 2009 \\
\hline Trimethyltin & mice & Mechanistic study & $\begin{array}{l}\text { Dey et al. 1997; Maurice et al. 1999; } \\
\text { Kassed, 2002 ; Kim, } 2007\end{array}$ \\
\hline Trimethyltin & rats & Behavioral analysis & Cohn and MacPhail 1996 \\
\hline Trimethyltin & rats & Mechanistic study & $\begin{array}{l}\text { Oconnell et al. 1994; OConnell et al. } \\
1996 \text { Tsutsumi, } 2002\end{array}$ \\
\hline Bisphenol & mice & $\begin{array}{l}\text { Developmental exposure } \\
\text { and behavioral analysis }\end{array}$ & Miyagawa et al. 2007 \\
\hline Bisphenol & rats & Mechanistic study & Poimenova et al. 2010 \\
\hline Paraben & rats & $\begin{array}{l}\text { Developmental exposure } \\
\text { and behavioral analysis }\end{array}$ & Kawaguchi et al. 2009 \\
\hline Salicylate & rats & $\begin{array}{l}\text { Developmental exposure } \\
\text { and behavioral analysis }\end{array}$ & Butcher et al. 1972 \\
\hline
\end{tabular}

Table 3. Literature lists for effects of environmental chemicals on memory and cognition in experimental animal models. 


\section{Conclusion}

ECSs are distributed widely and in increasing amounts over the world in the last few decades. ECSs exposure could occur through breastfeeding and hand-to-mouth activities in small children. In this review, epidemiological studies of children between ECSs exposure and neurodevelopmental disorders and experimental animal studies were focused. In our literature review, lead, methylmercury, pesticides, tobacco (cotinine), persistent organic pollutants such as PCBs, and environmental hormones such as bisphenol A and phthalates have been indicated association between neuronal disability and exposure levels in children. Children's brain and nervous system are vulnerable to adverse impacts from pollutants because they go through a long developmental process beginning shortly after conception and continuing through adolescence. This complex developmental process requires the precise coordination of cell growth and movement, and may be disrupted by even shortterm exposures to environmental contaminants if they occur at critical periods of development. This disruption can lead to neurodevelopmental deficits that may have an effect on the children's achievements and behaviors even though they do not result in a diagnosable disorder.

\section{Acknowledgment}

The authors thank Dr. Yoshika Kurokawa and Dr. Masami Ishido for their invaluable suggestions.

\section{References}

Aase H, Meyer A, Sagvolden T. 2006. Moment-to-moment dynamics of ADHD behaviour in South African children. Behav Brain Funct 2:11.

Abdel-Rahman A, Abou-Donia SM, El-Masry EM, Shetty AK, Abou-Donia MB. 2004. Stress and combined exposure to low doses of pyridostigmine bromide, deet, and permethrin produce neurochemical and neuropathological alterations in cerebral cortex, hippocampus, and cerebellum. Journal of Toxicology and Environmental Health-Part a-Current Issues 67(2):163-192.

Adhami VM, Husain R, Agarwal AK, Seth PK. 2000. Intrahippocampal cholinergic-rich transplants restore lead-induced deficits: a preliminary study in rats. Neurotoxicol Teratol 22(1):41-53.

Akahoshi E, Yoshimura S, Uruno S, Ishihara-Sugano M. 2009. Effect of dioxins on regulation of tyrosine hydroxylase gene expression by aryl hydrocarbon receptor: a neurotoxicology study. Environmental Health 8.

Albina ML, Belles M, Linares V, Sanchez DJ, Domingo JL. 2005. Restraint stress does not enhance the uranium-induced developmental and behavioral effects in the offspring of uranium-exposed male rats. Toxicology 215(1-2):69-79.

Aldridge JE, Levin ED, Seidler FJ, Slotkin TA. 2005. Developmental exposure of rats to chlorpyrifos leads to behavioral alterations in adulthood, involving serotonergic mechanisms and resembling animal models of depression. Environ Health Perspect 113(5):527-31. 
Alkondon M, Costa ACS, Radhakrishnan V, Aronstam RS, Albuquerque EX. 1990. Selective Blockade of Nmda-Activated Channel Currents May Be Implicated in LearningDeficits Caused by Lead. Febs Letters 261(1):124-130.

Allen AM, Dietz PM, Tong VT, England L, Prince CB. 2008. Prenatal smoking prevalence ascertained from two population-based data sources: birth certificates and PRAMS questionnaires, 2004. Public Health Rep 123(5):586-92.

al-Saleh I, Nester M, DeVol E, Shinwari N, Munchari L, al-Shahria S. 2001. Relationships between blood lead concentrations, intelligence, and academic achievement of Saudi Arabian schoolgirls. Int J Hyg Environ Health 204(2-3):165-74.

Altmann L, Weinsberg F, Sveinsson K, Lilienthal H, Wiegand H, Winneke G. 1993. Impairment of long-term potentiation and learning following chronic lead exposure. Toxicol Lett 66(1):105-12.

Ananth CV, Joseph KS, Oyelese Y, Demissie K, Vintzileos AM. 2005. Trends in preterm birth and perinatal mortality among singletons: United States, 1989 through 2000. Obstet Gynecol 105(5 Pt 1):1084-91.

Anderko L, Braun J, Auinger P. 2010. Contribution of tobacco smoke exposure to learning disabilities. J Obstet Gynecol Neonatal Nurs 39(1):111-7.

Andre SM, Markowski VP. 2006. Learning deficits expressed as delayed extinction of a conditioned running response following perinatal exposure to vinclozolin. Neurotoxicology and Teratology 28(4):482-488.

Aslan H, Songur A, Tunc AT, Ozen OA, Bas O, Yagmurca M, Turgut M, Sarsilmaz M, Kaplan S. 2006. Effects of formaldehyde exposure on granule cell number and volume of dentate gyrus: A histopathological and stereological study. Brain Research 1122:191-200.

Avila-Costa MR, Fortoul TI, Nino-Cabrera G, Colin-Barenque L, Bizarro-Nevares P, Gutierrez-Valdez AL, Ordonez-Librado JL, Rodriguez-Lara V, Mussali-Galante P, Diaz-Bech P and others. 2006. Hippocampal cell alterations induced by the inhalation of vanadium pentoxide $(\mathrm{V}(2) \mathrm{O}(5))$ promote memory deterioration. Neurotoxicology 27(6):1007-12.

Bandstra ES, Morrow CE, Mansoor E, Accornero VH. 2010. Prenatal drug exposure: infant and toddler outcomes. J Addict Dis 29(2):245-58.

Bareggi SR, Braida D, Pollera C, Bondiolotti G, Formentin E, Puricelli M, Poli G, Ponti W, Sala M. 2009. Effects of clioquinol on memory impairment and the neurochemical modifications induced by scrapie infection in golden hamsters. Brain Res 1280:195200.

Batstra L, Hadders-Algra M, Neeleman J. 2003. Effect of antenatal exposure to maternal smoking on behavioural problems and academic achievement in childhood: prospective evidence from a Dutch birth cohort. Early Human Development 75(12):21-33.

Begum AN, Yang F, Teng E, Hu S, Jones MR, Rosario ER, Beech W, Hudspeth B, Ubeda OJ, Cole GM and others. 2008. Use of copper and insulin-resistance to accelerate cognitive deficits and synaptic protein loss in a rat Abeta-infusion Alzheimer's disease model. J Alzheimers Dis 15(4):625-40.

Bellinger D, Leviton A, Waternaux C, Needleman H, Rabinowitz M. 1987. Longitudinal analyses of prenatal and postnatal lead exposure and early cognitive development. N Engl J Med 316(17):1037-43. 
Bellinger D, Sloman J, Leviton A, Rabinowitz M, Needleman HL, Waternaux C. 1991. Lowlevel lead exposure and children's cognitive function in the preschool years. Pediatrics 87(2):219-27.

Benetou-Marantidou A, Nakou S, Micheloyannis J. 1988. Neurobehavioral estimation of children with life-long increased lead exposure. Arch Environ Health 43(6):392-5.

Benetti F, Mello PB, Bonini JS, Monteiro S, Cammarota M, Izquierdo I. 2009. Early postnatal maternal deprivation in rats induces memory deficits in adult life that can be reversed by donepezil and galantamine. International Journal of Developmental Neuroscience 27(1):59-64.

Billauer-Haimovitch H, Slotkin TA, Dotan S, Langford R, Pinkas A, Yanai J. 2009. Reversal of chlorpyrifos neurobehavioral teratogenicity in mice by nicotine administration and neural stem cell transplantation. Behavioural Brain Research 205(2):499-504.

Binder JW, Roberts RJ. 1980. Carbon-Monoxide Intoxication in Children. Clinical Toxicology 16(3):287-295.

Bleecker ML, Ford DP, Lindgren KN, Hoese VM, Walsh KS, Vaughan CG. 2005. Differential effects of lead exposure on components of verbal memory. Occup Environ Med 62(3):181-7.

Boix J, Cauli O, Felipo V. 2010. Developmental exposure to polychlorinated biphenyls 52, 138 or 180 affects differentially learning or motor coordination in adult rats. Mechanisms involved. Neuroscience 167(4):994-1003.

Bolla-Wilson K, Bleecker ML. 1987. Neuropsychological impairment following inorganic arsenic exposure. J Occup Med 29(6):500-3.

Bowler RM, Lezak M, Booty A, Hartney C, Mergler D, Levin J, Zisman F. 2001. Neuropsychological dysfunction, mood disturbance, and emotional status of munitions workers. Appl Neuropsychol 8(2):74-90.

Bowler RM, Roels HA, Nakagawa S, Drezgic M, Diamond E, Park R, Koller W, Bowler RP, Mergler D, Bouchard M and others. 2007. Dose-effect relationships between manganese exposure and neurological, neuropsychological and pulmonary function in confined space bridge welders. Occup Environ Med 64(3):167-77.

Boyle CA, Boulet S, Schieve LA, Cohen RA, Blumberg SJ, Yeargin-Allsopp M, Visser S, Kogan MD. 2011. Trends in the prevalence of developmental disabilities in US children, 1997-2008. Pediatrics 127(6):1034-42.

Braun JM, Kahn RS, Froehlich T, Auinger P, Lanphear BP. 2006. Exposures to environmental toxicants and attention deficit hyperactivity disorder in U.S. children. Environ Health Perspect 114(12):1904-9.

Bressler JP, Goldstein GW. 1991. Mechanisms of lead neurotoxicity. Biochem Pharmacol 41(4):479-84.

Buchanan LH, Counter SA, Ortega F, Laurell G. 1999. Distortion product oto-acoustic emissions in Andean children and adults with chronic lead intoxication. Acta Otolaryngol 119(6):652-8.

Butcher RE, Vorhees CV, Kimmel CA. 1972. Leaning impairment from maternal salicylate treatment in rats. Nat New Biol 236(68):211-2.

Calas AG, Richard O, Meme S, Beloeil JC, Doan BT, Gefflaut T, Meme W, Crusio WE, Pichon J, Montecot C. 2008. Chronic exposure to glufosinate-ammonium induces spatial memory impairments, hippocampal MRI modifications and glutamine synthetase activation in mice. Neurotoxicology 29(4):740-7. 
Calderon J, Navarro ME, Jimenez-Capdeville ME, Santos-Diaz MA, Golden A, RodriguezLeyva I, Borja-Aburto V, Diaz-Barriga F. 2001. Exposure to arsenic and lead and neuropsychological development in Mexican children. Environ Res 85(2):69-76.

Canfield RL, Kreher DA, Cornwell C, Henderson CR, Jr. 2003. Low-level lead exposure, executive functioning, and learning in early childhood. Child Neuropsychol 9(1):35-53.

Capel ID, Pinnock MH, Dorrell HM, Williams DC, Grant EC. 1981. Comparison of concentrations of some trace, bulk, and toxic metals in the hair of normal and dyslexic children. Clin Chem 27(6):879-81.

Castillo CG, Montante M, Dufour L, Martinez ML, Jimenez-Capdeville ME. 2002. Behavioral effects of exposure to endosulfan and methyl parathion in adult rats. Neurotoxicol Teratol 24(6):797-804.

CDC, Services DoHaH, Prevention CfDCa, Health NCfE. 2009. Fourth National Report on Human Exposure to Environmental Chemicals. http://www.cdc.gov/exposurereport/pdf/FourthReport.pdf.

Centers for Disease Control and Prevention 2005. Blood lead levels--United States, 19992002. MMWR Morb Mortal Wkly Rep 54(20):513-516.

Chen Q, Niu Y, Zhang R, Guo H, Gao Y, Li Y, Liu R. 2010. The toxic influence of paraquat on hippocampus of mice: involvement of oxidative stress. Neurotoxicology 31(3):3106.

Chen YC, Guo YL, Hsu CC, Rogan WJ. 1992. Cognitive development of Yu-Cheng ("oil disease") children prenatally exposed to heat-degraded PCBs. JAMA 268(22):32138.

Chen YC, Yu ML, Rogan WJ, Gladen BC, Hsu CC. 1994. A 6-year follow-up of behavior and activity disorders in the Taiwan Yu-cheng children. Am J Public Health 84(3):41521.

Chengzhi C, Yan T, Shuqun C, Xuejun J, Youbin Q, Yinyin X, Qian T, Baijie T. 2011. New candidate proteins for benzo(a)pyrene-induced spatial learning and memory deficits. J Toxicol Sci 36(2):163-71.

Chiodo LM, Covington C, Sokol RJ, Hannigan JH, Jannise J, Ager J, Greenwald M, DelaneyBlack V. 2007. Blood lead levels and specific attention effects in young children. Neurotoxicol Teratol 29(5):538-46.

Christenson GN, Griffin JR, De Land PN. 1991. Validity of the dyslexia screener. Optom Vis Sci 68(4):275-81.

Christenson GN, Griffin JR, Taylor M. 2001. Failure of blue-tinted lenses to change reading scores of dyslexic individuals. Optometry 72(10):627-33.

Cohn J, MacPhail RC. 1996. Acute trimethyltin exposure produces nonspecific effects on learning in rats working under a multiple repeated acquisition and performance schedule. Neurotoxicol Teratol 18(1):99-111.

Cohn J, MacPhail RC. 1997. Chlorpyrifos produces selective learning deficits in rats working under a schedule of repeated acquisition and performance. J Pharmacol Exp Ther 283(1):312-20.

Comin D, Gazarini L, Zanoni JN, Milani H, de Oliveira RMW. 2010. Vitamin E improves learning performance and changes the expression of nitric oxide-producing neurons in the brains of diabetic rats. Behavioural Brain Research 210(1):38-45. 
Counter SA, Buchanan LH, Ortega F. 2005. Mercury levels in urine and hair of children in an Andean gold-mining settlement. Int J Occup Environ Health 11(2):132-7.

Counter SA, Buchanan LH, Ortega F. 2008. Zinc protoporphyrin levels, blood lead levels and neurocognitive deficits in Andean children with chronic lead exposure. Clin Biochem 41(1-2):41-7.

Curzon P, Anderson DJ, Nikkel AL, Fox GB, Gopalakrishnan M, Decker MW, Bitner RS. 2006. Antisense knockdown of the rat alpha7 nicotinic acetylcholine receptor produces spatial memory impairment. Neurosci Lett 410(1):15-9.

DeGarmo DS, Reid JB, Leve LD, Chamberlain P, Knutson JF. 2010. Patterns and Predictors of Growth in Divorced Fathers' Health Status and Substance Use. American Journal of Mens Health 4(1):60-70.

Deschamps D, Geraud C, Julien H, Baud FJ, Dally S. 2003. Memory one month after acute carbon monoxide intoxication: a prospective study. Occupational and Environmental Medicine 60(3):212-216.

Devesa SS, Blot WJ, Stone BJ, Miller BA, Tarone RE, Fraumeni JF, Jr. 1995. Recent cancer trends in the United States. J Natl Cancer Inst 87(3):175-82.

Dey PM, Polunas MA, Philbert MA, Reuhl KR. 1997. Altered expression of polysialylated NCAM in mouse hippocampus following trimethyltin administration. Neurotoxicology 18(3):633-643.

Dick FD, Bourne VJ, Semple SE, Fox HC, Miller BG, Deary IJ, Whalley LJ. 2010. Solvent exposure and cognitive ability at age 67: a follow-up study of the 1947 Scottish Mental Survey. Occupational and Environmental Medicine 67(6):401-407.

Dorner G, Plagemann A. 2002. DDT in human milk and mental capacities in children at school age: An additional view on PISA 2000. Neuroendocrinology Letters 23(56):427-431.

Dufault R, Schnoll R, Lukiw WJ, Leblanc B, Cornett C, Patrick L, Wallinga D, Gilbert SG, Crider R. 2009. Mercury exposure, nutritional deficiencies and metabolic disruptions may affect learning in children. Behav Brain Funct 5:44.

Eddins D, Petro A, Pollard N, Freedman JH, Levin ED. 2008. Mercury-induced cognitive impairment in metallothionein-1/2 null mice. Neurotoxicol Teratol 30(2):88-95.

Falluel-Morel A, Sokolowski K, Sisti HM, Zhou X, Shors TJ, Dicicco-Bloom E. 2007. Developmental mercury exposure elicits acute hippocampal cell death, reductions in neurogenesis, and severe learning deficits during puberty. J Neurochem 103(5):1968-81.

Fan G, Feng C, Li Y, Wang C, Yan J, Li W, Feng J, Shi X, Bi Y. 2009. Selection of nutrients for prevention or amelioration of lead-induced learning and memory impairment in rats. Ann Occup Hyg 53(4):341-51.

Fedotova YO, Ordyan NE. 2010. Effects of 8-OH-DPAT and NAN-190 on AnxiousDepressive-Like Behavior of Female Rats during the Estrous Cycle. Bulletin of Experimental Biology and Medicine 150(2):165-167.

Fergusson DM, Horwood LJ, Lynskey MT. 1997. Early dentine lead levels and educational outcomes at 18 years. J Child Psychol Psychiatry 38(4):471-8.

Fergusson DM, Horwood LJ. 1993. The effects of lead levels on the growth of word recognition in middle childhood. Int J Epidemiol 22(5):891-7.

Fisher A, Brandeis R, Karton I, Pittel Z, Gurwitz D, Haring R, Sapir M, Levy A, Heldman E. 1991. (+-)-cis-2-methyl-spiro(1,3-oxathiolane-5,3')quinuclidine, an M1 selective 
cholinergic agonist, attenuates cognitive dysfunctions in an animal model of Alzheimer's disease. J Pharmacol Exp Ther 257(1):392-403.

Flora SJ, Saxena G, Mehta A. 2007. Reversal of lead-induced neuronal apoptosis by chelation treatment in rats: role of reactive oxygen species and intracellular $\mathrm{Ca}(2+) . \mathrm{J}$ Pharmacol Exp Ther 322(1):108-16.

Fredriksson A, Archer T. 2004. Neurobehavioural deficits associated with apoptotic neurodegeneration and vulnerability for ADHD. Neurotox Res 6(6):435-56.

Freire C, Ramos R, Puertas R, Lopez-Espinosa MJ, Julvez J, Aguilera I, Cruz F, Fernandez MF, Sunyer J, Olea N. 2010. Association of traffic-related air pollution with cognitive development in children. J Epidemiol Community Health 64(3):223-8.

Froehlich TE, Lanphear BP, Auinger P, Hornung R, Epstein JN, Braun J, Kahn RS. 2009. Association of tobacco and lead exposures with attention-deficit/hyperactivity disorder. Pediatrics 124(6):e1054-63.

Gao MQ, Sun LG, Zhang TB, Teng Z, Liu S. 2005. [Effect of lead on learn and memory and release of intracellular free $\mathrm{Ca} 2+$ from calcium pool in dissociated mouse hippocampal neurons]. Wei Sheng Yan Jiu 34(4):400-2.

Garavan H, Morgan RE, Levitsky DA, Hermer-Vazquez L, Strupp BJ. 2000. Enduring effects of early lead exposure: evidence for a specific deficit in associative ability. Neurotoxicology and Teratology 22(2):151-164.

Garcia-Arenas G, Ramirez-Amaya V, Balderas I, Sandoval J, Escobar ML, Rios C, BermudezRattoni F. 2004. Cognitive deficits in adult rats by lead intoxication are related with regional specific inhibition of cNOS. Behav Brain Res 149(1):49-59.

Gilboa SM, Salemi JL, Nembhard WN, Fixler DE, Correa A. 2010. Mortality resulting from congenital heart disease among children and adults in the United States, 1999 to 2006. Circulation 122(22):2254-63.

Gong QH, Wu Q, Huang XN, Sun AS, Nie J, Shi JS. 2006. Protective effect of Ginkgo biloba leaf extract on learning and memory deficit induced by aluminum in model rats. Chin J Integr Med 12(1):37-41.

Griffin JR, Birch TF, Bateman GF, De Land PN. 1993. Dyslexia and visual perception: is there a relation? Optom Vis Sci 70(5):374-9.

Gronlund J, Nanto-Salonen K, Venetoklis J, Holmberg RL, Heinonen A, Stahlberg MR. 2006. Poor cognitive development and abdominal pain: Wilson's disease. Scand J Gastroenterol 41(3):361-4.

Gross MD, Tofanelli RA, Butzirus SM, Snodgrass EW. 1987. The effect of diets rich in and free from additives on the behavior of children with hyperkinetic and learning disorders. J Am Acad Child Adolesc Psychiatry 26(1):53-5.

Grossi C, Francese S, Casini A, Rosi MC, Luccarini I, Fiorentini A, Gabbiani C, Messori L, Moneti G, Casamenti F. 2009. Clioquinol decreases amyloid-beta burden and reduces working memory impairment in a transgenic mouse model of Alzheimer's disease. J Alzheimers Dis 17(2):423-40.

Guerrero AL, Dorado-Martinez C, Rodriguez A, Pedroza-Rios K, Borgonio-Perez G, RivasArancibia S. 1999. Effects of vitamin E on ozone-induced memory deficits and lipid peroxidation in rats. Neuroreport 10(8):1689-1692.

Guilarte TR, Chen MK. 2007. Manganese inhibits NMDA receptor channel function: implications to psychiatric and cognitive effects. Neurotoxicology 28(6):1147-52. 
Haider S, Shameem S, Ahmed SP, Perveen T, Haleem DJ. 2005. Repeated administration of lead decreases brain 5-HT metabolism and produces memory deficits in rats. Cell Mol Biol Lett 10(4):669-76.

Han F, Shioda N, Moriguchi S, Qin ZH, Fukunaga K. 2008. The vanadium (IV) compound rescues septo-hippocampal cholinergic neurons from neurodegeneration in olfactory bulbectomized mice. Neuroscience 151(3):671-9.

Han ST, Bhopale VM, Thom SR. 2007. Xanthine oxidoreductase and neurological sequelae of carbon monoxide poisoning. Toxicology Letters 170(2):111-115.

Hanninen H, Eskelinen L, Husman K, Nurminen M. 1976. Behavioral effects of long-term exposure to a mixture of organic solvents. Scand J Work Environ Health 2(4):24055.

Hellberg J. 1972. [Neurotoxic effects of methyl mercury on the squirrel monkey (Saimiri sciureus). Behavior and function disorders]. Nord Hyg Tidskr 53(1):5-10.

Hertz-Picciotto I, Bergman A, Fangstrom B, Rose M, Krakowiak P, Pessah I, Hansen R, Bennett DH. 2011. Polybrominated diphenyl ethers in relation to autism and developmental delay: a case-control study. Environ Health 10(1):1.

Houpert P, Bizot JC, Bussy C, Dhieux B, Lestaevel P, Gourmelon P, Paquet F. 2007. Comparison of the effects of enriched uranium and 137-cesium on the behaviour of rats after chronic exposure. Int J Radiat Biol 83(2):99-104.

Itoh A, Nitta A, Hirose M, Hasegawa T, Nabeshima T. 1997a. Effects of metrifonate on impairment of learning and dysfunction of cholinergic neuronal system in basal forebrain-lesioned rats. Behavioural Brain Research 83(1-2):165-167.

Itoh A, Nitta A, Katono Y, Usui M, Naruhashi K, Iida R, Hasegawa T, Nabeshima T. 1997b. Effects of metrifonate on memory impairment and cholinergic dysfunction in rats (vol 322, pg 11, 1997). European Journal of Pharmacology 332(1):113-113.

Ivanovic DM, Leiva BP, Perez HT, Inzunza NB, Almagia AF, Toro TD, Urrutia MS, Cervilla JO, Bosch EO. 2000. Long-term effects of severe undernutrition during the first year of life on brain development and learning in Chilean high-school graduates. Nutrition 16(11-12):1056-63.

Jevtovic-Todorovic V, Hartman RE, Izumi Y, Benshoff ND, Dikranian K, Zorumski CF, Olney JW, Wozniak DF. 2003. Early exposure to common anesthetic agents causes widespread neurodegeneration in the developing rat brain and persistent learning deficits. Journal of Neuroscience 23(3):876-882.

Johansson C, Castoldi AF, Onishchenko N, Manzo L, Vahter M, Ceccatelli S. 2007. Neurobehavioural and molecular changes induced by methylmercury exposure during development. Neurotox Res 11(3-4):241-60.

Johansson N, Fredriksson A, Eriksson P. 2008. Neonatal exposure to perfluorooctane sulfonate (PFOS) and perfluorooctanoic acid (PFOA) causes neurobehavioural defects in adult mice. Neurotoxicology 29(1):160-9.

Jolous-Jamshidi B, Cromwell HC, McFarland AM, Meserve LA. 2010. Perinatal exposure to polychlorinated biphenyls alters social behaviors in rats. Toxicol Lett 199(2):136-43.

Jusko TA, Henderson CR, Lanphear BP, Cory-Slechta DA, Parsons PJ, Canfield RL. 2008. Blood lead concentrations $<10 \mathrm{microg} / \mathrm{dL}$ and child intelligence at 6 years of age. Environ Health Perspect 116(2):243-8. 
Kalyva E. 2007. Prevalence and influences on self-reported smoking among adolescents with mild learning disabilities, attention deficit hyperactivity disorder, and their typically developing peers. J Intellect Disabil 11(3):267-79.

Kaneko N, Takada J, Yasui H, Sakurai H. 2006. Memory deficit in mice administered aluminum-maltolate complex. Biometals 19(1):83-9.

Kargoshaie AA, Najafi M, Akhlaghi M, Khazraie HR, Hekmatdoost A. 2009. The correlation between tonsil size and academic performance is not a direct one, but the results of various factors. Acta Otorhinolaryngol Ital 29(5):255-8.

Kassed CA, Willing AE, Garbuzova-Davis S, Sanberg PR, Pennypacker KR. 2002. Lack of NF-kappaB p50 exacerbates degeneration of hippocampal neurons after chemical exposure and impairs learning. Exp Neurol 176(2):277-88.

Katirci Y, Kandis H, Aslan S, Kirpinar I. 2011. Neuropsychiatric disorders and risk factors in carbon monoxide intoxication. Toxicology and Industrial Health 27(5):397-406.

Kawaguchi M, Irie K, Morohoshi K, Watanabe G, Taya K, Morita M, Kondo Y, Imai H, Himi T. 2009. Maternal isobutyl-paraben exposure alters anxiety and passive avoidance test performance in adult male rats. Neuroscience Research 65(2):136-40.

Keselyak NT, Simmer-Beck M, Bray KK, Gadbury-Amyot CC. 2007. Evaluation of an academic service-learning course on special needs patients for dental hygiene students: A qualitative study. Journal of Dental Education 71(3):378-392.

Kim MJ, Choi SJ, Lim ST, Kim HK, Heo HJ, Kim EK, Jun WJ, Cho HY, Kim YJ, Shin DH. 2007. Ferulic acid supplementation prevents trimethyltin-induced cognitive deficits in mice. Bioscience Biotechnology and Biochemistry 71(4):1063-1068.

Kim Y, Cho SC, Kim BN, Hong YC, Shin MS, Yoo HJ, Kim JW, Bhang SY. 2010. Association between blood lead levels $(<5 \mathrm{mug} / \mathrm{dL})$ and inattention-hyperactivity and neurocognitive profiles in school-aged Korean children. Sci Total Environ 408(23):5737-43.

Kofman O, Berger A, Massarwa A, Friedman A, Jaffar AA. 2006. Motor inhibition and learning impairments in school-aged children following exposure to organophosphate pesticides in infancy. Pediatr Res 60(1):88-92.

Kukla L, Hruba D, Tyrlik M. 2008. Maternal smoking during pregnancy, behavioral problems and school performances of their school-aged children. Cent Eur J Public Health 16(2):71-6.

Kumar MV, Desiraju T. 1992. EEG spectral power reduction and learning disability in rats exposed to lead through postnatal developing age. Indian J Physiol Pharmacol 36(1):15-20.

Kumar P, Kumar A. 2009. Effect of lycopene and epigallocatechin-3-gallate against 3nitropropionic acid induced cognitive dysfunction and glutathione depletion in rat: A novel nitric oxide mechanism. Food and Chemical Toxicology 47(10):2522-2530.

Landrigan PJ, Trasande L, Thorpe LE, Gwynn C, Lioy PJ, D'Alton ME, Lipkind HS, Swanson J, Wadhwa PD, Clark EB and others. 2006. The National Children's Study: a 21-year prospective study of 100,000 American children. Pediatrics 118(5):2173-86.

Lanphear BP, Hornung R, Khoury J, Yolton K, Baghurst P, Bellinger DC, Canfield RL, Dietrich KN, Bornschein R, Greene T and others. 2005. Low-level environmental lead exposure and children's intellectual function: an international pooled analysis. Environ Health Perspect 113(7):894-9. 
Larson SA, Lakin KC, Anderson L, Kwak Lee N, Anderson D. 2001. Prevalence of mental retardation and developmental disabilities: estimates from the 1994/1995 national health interview survey disability supplements. Am J Ment Retard 106(3):231-52.

Lassen K, Oei TPS. 1998. Effects of maternal cigarette smoking during pregnancy on longterm physical and cognitive parameters of child development. Addictive Behaviors 23(5):635-653.

Lee DH, Jacobs DR, Porta M. 2007. Association of serum concentrations of persistent organic pollutants with the prevalence of learning disability and attention deficit disorder. J Epidemiol Community Health 61(7):591-6.

Levin ED, Addy N, Baruah A, Elias A, Christopher NC, Seidler FJ, Slotkin TA. 2002. Prenatal chlorpyrifos exposure in rats causes persistent behavioral alterations. Neurotoxicology and Teratology 24(6):733-741.

Levin ED, Perkins A, Brotherton T, Qazi M, Berez C, Montalvo-Ortiz J, Davis K, Williams P, Christopher NC. 2009. Chronic underactivity of medial frontal cortical beta2containing nicotinic receptors increases clozapine-induced working memory impairment in female rats. Prog Neuropsychopharmacol Biol Psychiatry 33(2):296302.

Levin ED, Timofeeva OA, Yang LW, Petro A, Ryde IT, Wrench N, Seidler FJ, Slotkin TA. 2010. Early postnatal parathion exposure in rats causes sex-selective cognitive impairment and neurotransmitter defects which emerge in aging. Behavioural Brain Research 208(2):319-327.

Levin ED, Wilkerson A, Jones JP, Christopher NC, Briggs SJ. 1996. Prenatal nicotine effects on memory in rats: Pharmacological and behavioral challenges. Developmental Brain Research 97(2):207-215.

Leviton A, Bellinger D, Allred EN, Rabinowitz M, Needleman H, Schoenbaum S. 1993. Preand postnatal low-level lead exposure and children's dysfunction in school. Environ Res 60(1):30-43.

Liang K, Poytress BS, Chen YL, Leslie FM, Weinberger NM, Metherate R. 2006. Neonatal nicotine exposure impairs nicotinic enhancement of central auditory processing and auditory learning in adult rats. European Journal of Neuroscience 24(3):857866.

Lin CS, Tao PL, Jong YJ, Chen WF, Yang CH, Huang LT, Chao CF, Yang SN. 2009. Prenatal morphine alters the synaptic complex of postsynaptic density 95 with N-methyl-Daspartate receptor subunit in hippocampal CA1 subregion of rat offspring leading to long-term cognitive deficits. Neuroscience 158(4):1326-37.

Lin KC, Guo NW, Tsai PC, Yang CY, Guo YLL. 2008. Neurocognitive changes among elderly exposed to PCBs/PCDFs in Taiwan. Environmental Health Perspectives 116(2):184-189.

Liu Y, Ye Z, Yang H, Zhou L, Fan D, He S, Chui D. 2010. Disturbances of soluble Nethylmaleimide-sensitive factor attachment proteins in hippocampal synaptosomes contribute to cognitive impairment after repetitive formaldehyde inhalation in male rats. Neuroscience 169(3):1248-54.

LoSasso GL, Rapport LJ, Axelrod BN. 2001. Neuropsychological symptoms associated with low-level exposure to solvents and (meth)acrylates among nail technicians. Neuropsychiatry Neuropsychology and Behavioral Neurology 14(3):183-189. 
LoSasso GL, Rapport LJ, Axelrod TN, Whitman RD. 2002. Neurocognitive sequelae of exposure to organic solvents and (meth)acrylates among nail-studio technicians. Neuropsychiatry Neuropsychology and Behavioral Neurology 15(1):44-55.

Lu J, Wu DM, Zheng YL, Sun DX, Hu B, Shan Q, Zhang ZF, Fan SH. 2009. Trace amounts of copper exacerbate beta amyloid-induced neurotoxicity in the cholesterol-fed mice through TNF-mediated inflammatory pathway. Brain Behavior and Immunity 23(2):193-203.

Lu J, Zheng YL, Wu DM, Sun DX, Shan Q, Fan SH. 2006. Trace amounts of copper induce neurotoxicity in the cholesterol-fed mice through apoptosis. Febs Letters 580(2829):6730-6740.

Lyngbye T, Hansen ON, Trillingsgaard A, Beese I, Grandjean P. 1990. Learning disabilities in children: significance of low-level lead-exposure and confounding factors. Acta Paediatr Scand 79(3):352-60.

Madrid PA, Sinclair H, Bankston AQ, Overholt S, Brito A, Domnitz R, Grant R. 2008. Building integrated mental health and medical programs for vulnerable populations post-disaster: connecting children and families to a medical home. Prehosp Disaster Med 23(4):314-21.

Mahmoudian T, Modaresi M, Zarei A, Poursafa P, Kelishadi R. 2009. Blood lead levels in children with neurological disorders: a single centre preliminary study. Zhongguo Dang Dai Er Ke Za Zhi 11(11):873-6.

Malik G, Tagiyeva N, Aucott L, McNeill G, Turner SW. 2011. Changing trends in asthma in 9-12 year olds between 1964 and 2009. Arch Dis Child 96(3):227-31.

Mao X, Zhang L, Xia Q, Sun Z, Zhao X, Cai H, Yang X, Xia Z, Tang Y. 2008. Vanadiumenriched chickpea sprout ameliorated hyperglycemia and impaired memory in streptozotocin-induced diabetes rats. Biometals 21(5):563-70.

Marcus MM, Jardemark KE, Wadenberg ML, Langlois X, Hertel P, Svensson TH. 2005. Combined alpha2 and D2/3 receptor blockade enhances cortical glutamatergic transmission and reverses cognitive impairment in the rat. Int $\mathrm{J}$ Neuropsychopharmacol 8(3):315-27.

Marshall R, Barkess-Jones L, Sivayoham S. 1995. An outbreak of scabies in a school for children with learning disabilities. Commun Dis Rep CDR Rev 5(6):R90-2.

Martinez-Finley EJ, Ali AM, Allan AM. 2009. Learning deficits in C57BL/6J mice following perinatal arsenic exposure: consequence of lower corticosterone receptor levels? Pharmacol Biochem Behav 94(2):271-7.

Matsumoto T, Kamijo A, Yamaguchi A, Iseki E, Hirayasu Y. 2005. Childhood histories of attention-deficit hyperactivity disorders in Japanese methamphetamine and inhalant abusers: preliminary report. Psychiatry Clin Neurosci 59(1):102-5.

Maurice T, Phan VL, Noda Y, Yamada K, Privat A, Nabeshima T. 1999. The attenuation of learning impairments induced after exposure to $\mathrm{CO}$ or trimethyltin in mice by sigma (sigma) receptor ligands involves both sigma(1) and sigma(2) sites. British Journal of Pharmacology 127(2):335-342.

McMichael AJ, Baghurst PA, Wigg NR, Vimpani GV, Robertson EF, Roberts RJ. 1988. Port Pirie Cohort Study: environmental exposure to lead and children's abilities at the age of four years. N Engl J Med 319(8):468-75.

Mendelsohn AL, Dreyer BP, Fierman AH, Rosen CM, Legano LA, Kruger HA, Lim SW, Courtlandt CD. 1998. Low-level lead exposure and behavior in early childhood. Pediatrics 101(3):E10. 
Meunier J, Ieni J, Maurice T. 2006. Antiamnesic and neuroprotective effects of donepezil against learning impairments induced in mice by exposure to carbon monoxide gas. J Pharmacol Exp Ther 317(3):1307-19.

Minder B, Das-Smaal EA, Brand EF, Orlebeke JF. 1994. Exposure to lead and specific attentional problems in schoolchildren. J Learn Disabil 27(6):393-9.

Minder B, Das-Smaal EA, Orlebeke JF. 1998. Cognition in children does not suffer from very low lead exposure. J Learn Disabil 31(5):494-502.

Miyagawa K, Narita M, Akama H, Suzuki T. 2007. Memory impairment associated with a dysfunction of the hippocampal cholinergic system induced by prenatal and neonatal exposures to bisphenol-A. Neurosci Lett 418(3):236-41.

Moen BE, Riise T, Haga EM, Fossan GO. 1990. Reduced Performance in Tests of Memory and Visual Abstraction in Seamen Exposed to Industrial Solvents. Acta Psychiatrica Scandinavica 81(2):114-119.

Molina BSG, Pelham WE. 2001. Substance use, substance abuse, and LD among adolescents with a childhood history of ADHD. Journal of Learning Disabilities 34(4):333-+.

Momcilovic B. 1999. A case report of acute human molybdenum toxicity from a dietary molybdenum supplement--a new member of the "Lucor metallicum" family. Arh Hig Rada Toksikol 50(3):289-97.

Montgomery KS, Mackey J, Thuett K, Ginestra S, Bizon JL, Abbott LC. 2008. Chronic, lowdose prenatal exposure to methylmercury impairs motor and mnemonic function in adult C57/B6 mice. Behav Brain Res 191(1):55-61.

Morrow LA, Robin N, Hodgson MJ, Kamis H. 1992. Assessment of Attention and Memory Efficiency in Persons with Solvent Neurotoxicity. Neuropsychologia 30(10):911-922.

Morrow LA, Stein L, Bagovich GR, Condray R, Scott A. 2001. Neuropsychological assessment, depression, and past exposure to organic solvents. Appl Neuropsychol 8(2):65-73.

Morton WE, Caron GA. 1989. Encephalopathy: an uncommon manifestation of workplace arsenic poisoning? Am J Ind Med 15(1):1-5.

Moss HB, Talagala SL, Kirisci L. 1997. Phosphorus-31 magnetic resonance brain spectroscopy of children at risk for a substance use disorder: Preliminary results. Psychiatry Research-Neuroimaging 76(2-3):101-112.

Najman JM, Aird R, Bor W, O'Callaghan M, Williams GM, Shuttlewood GJ. 2004. The generational transmission of socioeconomic inequalities in child cognitive development and emotional health. Soc Sci Med 58(6):1147-58.

Nerobkova LN, Voronina TA. 1988. [Disordered learning and sleep structure in rats with a cobalt-induced epileptogenic focus in the sensorimotor cortex]. Biull Eksp Biol Med 105(2):139-42.

Nigg JT, Knottnerus GM, Martel MM, Nikolas M, Cavanagh K, Karmaus W, Rappley MD. 2008. Low blood lead levels associated with clinically diagnosed attentiondeficit/hyperactivity disorder and mediated by weak cognitive control. Biol Psychiatry 63(3):325-31.

Nishio H, Kasuga S, Ushijima M, Harada Y. 2001. Prenatal stress and postnatal development of neonatal rats sex-dependent effects on emotional behavior and learning ability of neonatal rats. International Journal of Developmental Neuroscience 19(1):37-45.

Obernier JA, White AM, Swartzwelder HS, Crews FT. 2002. Cognitive deficits and CNS damage after a 4-day binge ethanol exposure in rats. Pharmacol Biochem Behav 72(3):521-32. 
O'Brien LM, Mervis CB, Holbrook CR, Bruner JL, Smith NH, McNally N, McClimment MC, Gozal D. 2004. Neurobehavioral correlates of sleep-disordered breathing in children. Journal of Sleep Research 13(2):165-172.

O'Callaghan FV, Al Mamun A, O'Callaghan M, Alati R, Williams GM, Najman JM. 2010. Is smoking in pregnancy an independent predictor of academic difficulties at 14years of age? A birth cohort study. Early Human Development 86(2):71-6.

Oconnell A, Earley B, Leonard BE. 1994. The Neuroprotective Effect of Tacrine on Trimethyltin Induced Memory and Muscarinic Receptor Dysfunction in the Rat. Neurochemistry International 25(6):555-566.

OConnell AW, Earley B, Leonard E. 1996. The sigma ligand JO 1784 prevents trimethyltininduced behavioural and sigma-receptor dysfunction in the rat. Pharmacology \& Toxicology 78(5):296-302.

Palumbo G, Bacchi S, Coppolino MF, Pantaleoni GC. 2001. Neurochemical and behavioural effects of chronic aldicarb administration in rats. Pharmacology \& Toxicology 89(5):249-254.

Palumbo ML, Fosser NS, Rios H, Zubilete MAZ, Guelman LR, Cremaschi GA, Genaro AM. 2007. Loss of hippocampal neuronal nitric oxide synthase contributes to the stressrelated deficit in learning and memory. Journal of Neurochemistry 102(1):261-274.

Paul V, Balasubramaniam E, Kazi M. 1994. The neurobehavioural toxicity of endosulfan in rats: a serotonergic involvement in learning impairment. European Journal of Pharmacology 270(1):1-7.

Paul V, Reddy L, Ekambaram P. 2003. Prevention of picrotoxin convulsions-induced learning and memory impairment by nitric oxide increasing dose of L-arginine in rats. Pharmacology Biochemistry and Behavior 75(2):329-334.

Paz R, Barsness B, Martenson T, Tanner D, Allan AM. 2007. Behavioral teratogenicity induced by nonforced maternal nicotine consumption. Neuropsychopharmacology 32(3):693-9.

Petry C, Pereira MU, Pitrez PM, Jones MH, Stein RT. 2008. The prevalence of symptoms of sleep-disordered breathing in Brazilian schoolchildren. J Pediatr (Rio J) 84(2):123-9.

Pfeiffer CC, Braverman ER. 1982. Zinc, the brain and behavior. Biol Psychiatry 17(4):513-32.

Piedrafita B, Erceg S, Cauli O, Monfort P, Felipo V. 2008. Developmental exposure to polychlorinated biphenyls PCB153 or PCB126 impairs learning ability in young but not in adult rats. European Journal of Neuroscience 27(1):177-182.

Pihl RO, Parkes M. 1977. Hair element content in learning disabled children. Science 198(4313):204-6.

Piikivi L, Hanninen H. 1989. Subjective symptoms and psychological performance of chlorine-alkali workers. Scand J Work Environ Health 15(1):69-74.

Poimenova A, Markaki E, Rahiotis C, Kitraki E. 2010. Corticosterone-regulated actions in the rat brain are affected by perinatal exposure to low dose of bisphenol A. Neuroscience 167(3):741-9.

Post AM, Wultsch T, Popp S, Painsipp E, Wetzstein H, Kittel-Schneider S, Sontag TA, Lesch $\mathrm{KP}$, Reif A. 2011. The COGITAT holeboard system as a valuable tool to assess learning, memory and activity in mice. Behavioural Brain Research 220(1):152-158.

Prevention. CfDCa. 2005. Blood lead levels--United States, 1999-2002. MMWR Morb Mortal Wkly Rep 54(20):513-6. 
Quinn JF, Harris CJ, Cobb KE, Domes C, Ralle M, Brewer G, Wadsworth TL. 2010. A copperlowering strategy attenuates amyloid pathology in a transgenic mouse model of Alzheimer's disease. J Alzheimers Dis 21(3):903-14.

Rabinowitz MB, Wang JD, Soong WT. 1992. Children's classroom behavior and lead in Taiwan. Bull Environ Contam Toxicol 48(2):282-8.

Railey AM, Groeber CM, Flinn JM. 2011. The effect of metals on spatial memory in a transgenic mouse model of Alzheimer's disease. J Alzheimers Dis 24(2):375-81.

Rauh VA, Garfinkel R, Perera FP, Andrews HF, Hoepner L, Barr DB, Whitehead R, Tang D, Whyatt RW. 2006. Impact of prenatal chlorpyrifos exposure on neurodevelopment in the first 3 years of life among inner-city children. Pediatrics 118(6):e1845-59.

Reddy DS, Kulkarni SK. 1998. Possible role of nitric oxide in the nootropic and antiamnesic effects of neurosteroids on aging- and dizocilpine-induced learning impairment. Brain Research 799(2):215-229.

Redwood L, Bernard S, Brown D. 2001. Predicted mercury concentrations in hair from infant immunizations: cause for concern. Neurotoxicology 22(5):691-7.

Reidy TJ, Bolter JF, Cone JE. 1994. Neuropsychological sequelae of methyl bromide: a case study. Brain Inj 8(1):83-93.

Revest JM, Dupret D, Koehl M, Funk-Reiter C, Grosjean N, Piazza PV, Abrous DN. 2009. Adult hippocampal neurogenesis is involved in anxiety-related behaviors. Molecular Psychiatry 14(10):959-967.

Richter E, Friesenegger S, Engl J, Tricker AR. 2000. Use of precision-cut tissue slices in organ culture to study metabolism of 4-(methylnitrosamino)-1-(3-pyridyl)-1-butanone (NNK) and 4-(methylnitrosamino)-1-(3-pyridyl)-1-butanol (NNAL) by hamster lung, liver and kidney. Toxicology 144(1-3):83-91.

Rimland B, Larson GE. 1983. Hair mineral analysis and behavior: an analysis of 51 studies. J Learn Disabil 16(5):279-85.

Ris MD, Dietrich KN, Succop PA, Berger OG, Bornschein RL. 2004. Early exposure to lead and neuropsychological outcome in adolescence. J Int Neuropsychol Soc 10(2):26170.

Robertson SB, Jackson C. 1996. Initiation of cigarette smoking among children with and without learning disabilities. Journal of Developmental and Behavioral Pediatrics 17(4):248-252.

Robison LL, Buckley JD, Bunin G. 1995. Assessment of environmental and genetic factors in the etiology of childhood cancers: the Childrens Cancer Group epidemiology program. Environ Health Perspect 103 Suppl 6:111-6.

Rodriguez VM, Carrizales L, Jimenez-Capdeville ME, Dufour L, Giordano M. 2001. The effects of sodium arsenite exposure on behavioral parameters in the rat. Brain Res Bull 55(2):301-8.

Rodriguez VM, Carrizales L, Mendoza MS, Fajardo OR, Giordano M. 2002. Effects of sodium arsenite exposure on development and behavior in the rat. Neurotoxicology and Teratology 24(6):743-750.

Roegge CS, Schantz SL. 2006. Motor function following developmental exposure to PCBS and/or MEHG. Neurotoxicol Teratol 28(2):260-77.

Rowland AS, Lesesne CA, Abramowitz AJ. 2002. The epidemiology of attentiondeficit/hyperactivity disorder (ADHD): a public health view. Ment Retard Dev Disabil Res Rev 8(3):162-70. 
Rueda N, Florez J, Martinez-Cue C. 2008. Effects of chronic administration of SGS-111 during adulthood and during the pre- and post-natal periods on the cognitive deficits of Ts65Dn mice, a model of Down syndrome. Behavioural Brain Research 188(2):355-367.

Ryan CM, Morrow LA, Hodgson M. 1988. Cacosmia and neurobehavioral dysfunction associated with occupational exposure to mixtures of organic solvents. Am J Psychiatry 145(11):1442-5.

Sakamoto M, Kakita A, de Oliveira RB, Sheng Pan H, Takahashi H. 2004. Dose-dependent effects of methylmercury administered during neonatal brain spurt in rats. Brain Res Dev Brain Res 152(2):171-6.

Sakamoto M, Kakita A, Wakabayashi K, Takahashi H, Nakano A, Akagi H. 2002. Evaluation of changes in methylmercury accumulation in the developing rat brain and its effects: a study with consecutive and moderate dose exposure throughout gestation and lactation periods. Brain Research 949(1-2):51-59.

Sanchez DJ, Belles M, Albina ML, Gomez M, Linares V, Domingo JL. 2006. Exposure of pregnant rats to uranium and restraint stress: Effects on postnatal development and behavior of the offspring. Toxicology 228(2-3):323-332.

Sandberg DE, Vena JE, Weiner J, Beehler GP, Swanson M, Meyer-Bahlburg HF. 2003. Hormonally active agents in the environment and children's behavior: assessing effects on children's gender-dimorphic outcomes. Epidemiology 14(2):148-54.

Schantz SL, Gasior DM, Polverejan E, McCaffrey RJ, Sweeney AM, Humphrey HEB, Gardiner JC. 2001. Impairments of memory and learning in older adults exposed to polychlorinated biphenyls via consumption of great lakes fish. Environmental Health Perspectives 109(6):605-611.

Schechter CB. 1999. Re: Brain and other central nervous system cancers: recent trends in incidence and mortality. J Natl Cancer Inst 91(23):2050-1.

Schettler T. 2001. Toxic threats to neurologic development of children. Environ Health Perspect 109 Suppl 6:813-6.

Schnaas L, Rothenberg SJ, Flores MF, Martinez S, Hernandez C, Osorio E, Velasco SR, Perroni E. 2006. Reduced intellectual development in children with prenatal lead exposure. Environ Health Perspect 114(5):791-7.

Schrauzer GN, Shrestha KP, Flores-Arce MF. 1992. Lithium in scalp hair of adults, students, and violent criminals. Effects of supplementation and evidence for interactions of lithium with vitamin B12 and with other trace elements. Biol Trace Elem Res 34(2):161-76.

Schwartz BS, Chen S, Caffo B, Stewart WF, Bolla KI, Yousem D, Davatzikos C. 2007. Relations of brain volumes with cognitive function in males 45 years and older with past lead exposure. Neuroimage 37(2):633-41.

Schwartz BS, Stewart WF, Bolla KI, Simon PD, Bandeen-Roche K, Gordon PB, Links JM, Todd AC. 2000. Past adult lead exposure is associated with longitudinal decline in cognitive function. Neurology 55(8):1144-50.

Sethi P, Jyoti A, Hussain E, Sharma D. 2009. Curcumin attenuates aluminium-induced functional neurotoxicity in rats. Pharmacol Biochem Behav 93(1):31-9.

Sharma M, Purdy SC, Kelly AS. 2009. Comorbidity of auditory processing, language, and reading disorders. J Speech Lang Hear Res 52(3):706-22. 
Sheng L, Ding X, Ferguson M, McCallister M, Rhoades R, Maguire M, Ramesh A, Aschner M, Campbell D, Levitt P and others. 2010. Prenatal polycyclic aromatic hydrocarbon exposure leads to behavioral deficits and downregulation of receptor tyrosine kinase, MET. Toxicol Sci 118(2):625-34.

Shih RA, Glass TA, Bandeen-Roche K, Carlson MC, Bolla KI, Todd AC, Schwartz BS. 2006. Environmental lead exposure and cognitive function in community-dwelling older adults. Neurology 67(9):1556-62.

Simons TJ. 1993. Lead-calcium interactions in cellular lead toxicity. Neurotoxicology 14(23):77-85.

Sinha C, Seth K, Islam F, Chaturvedi RK, Shukla S, Mathur N, Srivastava N, Agrawal AK. 2006. Behavioral and neurochemical effects induced by pyrethroid-based mosquito repellent exposure in rat offsprings during prenatal and early postnatal period. Neurotoxicol Teratol 28(4):472-81.

Smith RC, Lindenmayer JP, Davis JM, Cornwell J, Noth K, Gupta S, Sershen H, Lajtha A. 2009. Cognitive and antismoking effects of varenicline in patients with schizophrenia or schizoaffective disorder. Schizophr Res 110(1-3):149-55.

Sparks DL, Schreurs BG. 2003. Trace amounts of copper in water induce beta-amyloid plaques and learning deficits in a rabbit model of Alzheimer's disease (vol 100, pg 11065, 2003). Proceedings of the National Academy of Sciences of the United States of America 100(20):11816-11816.

Spowart-Manning L, van der Staay FJ. 2005. Spatial discrimination deficits by excitotoxic lesions in the Morris water escape task. Behavioural Brain Research 156(2):269-276.

Srivastava AK, Gupta BN, Bihari V, Mathur N, Srivastava LP, Pangtey BS, Bharti RS, Kumar P. 2000. Clinical, biochemical and neurobehavioural studies of workers engaged in the manufacture of quinalphos. Food Chem Toxicol 38(1):65-9.

Stephens R, Spurgeon A, Calvert IA, Beach J, Levy LS, Berry H, Harrington JM. 1995. Neuropsychological effects of long-term exposure to organophosphates in sheep dip. Lancet 345(8958):1135-9.

Stewart PW, Sargent DM, Reihman J, Gump BB, Lonky E, Darvill T, Hicks H, Pagano J. 2006. Response inhibition during Differential Reinforcement of Low Rates (DRL) schedules may be sensitive to low-level polychlorinated biphenyl, methylmercury, and lead exposure in children. Environ Health Perspect 114(12):1923-9.

Stokes L, Letz R, Gerr F, Kolczak M, McNeill FE, Chettle DR, Kaye WE. 1998. Neurotoxicity in young adults 20 years after childhood exposure to lead: the Bunker Hill experience. Occup Environ Med 55(8):507-16.

Stollery BT, Flindt ML. 1988. Memory sequelae of solvent intoxication. Scand J Work Environ Health 14(1):45-8.

Sun SL, Ma GY, Li HB, Zhu YB, Dong HM, Xu XH. 2005. Effect of naloxone on aluminuminduced learning and memory impairment in rats. Neurology India 53(1):79-82.

Supriyadi E, Widjajanto PH, Purwanto I, Cloos J, Veerman AJ, Sutaryo S. 2011. Incidence of childhood leukemia in Yogyakarta, Indonesia, 1998-2009. Pediatr Blood Cancer 57(4):588-93.

Surkan PJ, Zhang A, Trachtenberg F, Daniel DB, McKinlay S, Bellinger DC. 2007. Neuropsychological function in children with blood lead levels $<10$ microg/dL. Neurotoxicology 28(6):1170-7. 
Tang HW, Liang YX, Hu XH. 1994. Effects of low level lead exposure on behavior of young rats. Zhongguo Yao Li Xue Bao 15(4):316-9.

Thom SR, Bhopale VM, Fisher D, Zhang J, Gimotty P. 2004. Delayed neuropathology after carbon monoxide poisoning is immune-mediated. Proceedings of the National Academy of Sciences of the United States of America 101(37):13660-13665.

Thomson GO, Raab GM, Hepburn WS, Hunter R, Fulton M, Laxen DP. 1989. Blood-lead levels and children's behaviour--results from the Edinburgh Lead Study. J Child Psychol Psychiatry 30(4):515-28.

Timofeeva OA, Roegge CS, Seidler FJ, Slotkin TA, Levin ED. 2008. Persistent cognitive alterations in rats after early postnatal exposure to low doses of the organophosphate pesticide, diazinon. Neurotoxicology and Teratology 30(1):38-45.

Tong S, Baghurst P, McMichael A, Sawyer M, Mudge J. 1996. Lifetime exposure to environmental lead and children's intelligence at 11-13 years: the Port Pirie cohort study. BMJ 312(7046):1569-75.

Tong ZQ, Zhang JL, Luo WH, Wang WS, Li FX, Li H, Luo HJ, Lu J, Zhou JN, Wan Y and others. 2011. Urine formaldehyde level is inversely correlated to mini mental state examination scores in senile dementia. Neurobiology of Aging 32(1):31-41.

Tsutsumi S, Akaike M, Arimitsu H, Imai H, Kato N. 2002. Circulating corticosterone alters the rate of neuropathological and behavioral changes induced by trimethyltin in rats. Experimental Neurology 173(1):86-94.

Tulve NS, Jones PA, Nishioka MG, Fortmann RC, Croghan CW, Zhou JY, Fraser A, Cavel C, Friedman W. 2006. Pesticide measurements from the first national environmental health survey of child care centers using a multi-residue GC/MS analysis method. Environ Sci Technol 40(20):6269-74.

Tuthill RW. 1996. Hair lead levels related to children's classroom attention-deficit behavior. Arch Environ Health 51(3):214-20.

Uzun N, Kendirli Y. 2005. Clinical, socio-demographic, neurophysiological and neuropsychiatric evaluation of children with volatile substance addiction. Child Care Health Dev 31(4):425-32.

Valent F, Pisa F, Mariuz M, Horvat M, Gibicar D, Fajon V, Mazej D, Daris F, Barbone F. 2011. [Fetal and perinatal exposure to mercury and selenium: baseline evaluation of a cohort of children in Friuli Venezia Giulia, Italy]. Epidemiol Prev 35(1):33-42.

Valkusz Z, Nagyeri G, Radacs M, Ocsko T, Hausinger P, Laszlo M, Laszlo FA, Juhasz A, Julesz J, Palfoldi R and others. 2011. Further analysis of behavioral and endocrine consequences of chronic exposure of male Wistar rats to subtoxic doses of endocrine disruptor chlorobenzenes. Physiology \& Behavior 103(5):421-430.

van den Hazel P, Zuurbier M, Babisch W, Bartonova A, Bistrup ML, Bolte G, Busby C, Butter M, Ceccatelli S, Fucic A and others. 2006. Today's epidemics in children: possible relations to environmental pollution and suggested preventive measures. Acta Paediatr Suppl 95(453):18-25.

van Elderen SG, de Roos A, de Craen AJ, Westendorp RG, Blauw GJ, Jukema JW, Bollen EL, Middelkoop HA, van Buchem MA, van der Grond J. 2010. Progression of brain atrophy and cognitive decline in diabetes mellitus: a 3-year follow-up. Neurology 75(11):997-1002.

van Wijngaarden E, Campbell JR, Cory-Slechta DA. 2009. Bone lead levels are associated with measures of memory impairment in older adults. Neurotoxicology 30(4):57280 . 
Vazquez A, Pena de Ortiz S. 2004. Lead $(\mathrm{Pb}(+2))$ impairs long-term memory and blocks learning-induced increases in hippocampal protein kinase $\mathrm{C}$ activity. Toxicol Appl Pharmacol 200(1):27-39.

Verma SK, Raheja G, Gill KD. 2009. Role of muscarinic signal transduction and CREB phosphorylation in dichlorvos-induced memory deficits in rats: An acetylcholine independent mechanism. Toxicology 256(3):175-182.

Vicente E, Boer M, Leite M, Silva M, Tramontina F, Porciuncula L, Dalmaz C, Goncalves CA. 2004. Cerebrospinal fluid S100B increases reversibly in neonates of methyl mercury-intoxicated pregnant rats. Neurotoxicology 25(5):771-7.

Walhovd KB, Moe V, Slinning K, Due-Tonnessen P, Bjornerud A, Dale AM, van der Kouwe A, Quinn BT, Kosofsky B, Greve D and others. 2007. Volumetric cerebral characteristics of children exposed to opiates and other substances in utero. Neuroimage 36(4):1331-44.

Wang CL, Chuang HY, Ho CK, Yang CY, Tsai JL, Wu TS, Wu TN. 2002. Relationship between blood lead concentrations and learning achievement among primary school children in Taiwan. Environ Res 89(1):12-8.

Wang HL, Chen XT, Yang B, Ma FL, Wang S, Tang ML, Hao MG, Ruan DY. 2008. Casecontrol study of blood lead levels and attention deficit hyperactivity disorder in Chinese children. Environ Health Perspect 116(10):1401-6.

Wang P, Zeng T, Zhang CL, Gao XC, Liu Z, Xie KQ, Chi ZF. 2009a. Lipid Peroxidation was Involved in the Memory Impairment of Carbon Monoxide-induced Delayed Neuron Damage. Neurochemical Research 34(7):1293-1298.

Wang Y, Browne DC, Petras H, Stuart EA, Wagner FA, Lambert SF, Kellam SG, Ialongo NS. $2009 \mathrm{~b}$. Depressed mood and the effect of two universal first grade preventive interventions on survival to the first tobacco cigarette smoked among urban youth. Drug and Alcohol Dependence 100(3):194-203.

Woodruff-Pak DS, Agelan A, Del Valle L. 2007. A rabbit model of Alzheimer's disease: Valid at neuropathological, cognitive, and therapeutic levels. Journal of Alzheimers Disease 11(3):371-383.

Xu X, Nembhard WN, Kan H, Kearney G, Zhang ZJ, Talbott EO. 2011. Urinary trichlorophenol levels and increased risk of attention deficit hyperactivity disorder among US school-aged children. Occup Environ Med 68(8):557-61.

Yang Y, Ma Y, Ni L, Zhao S, Li L, Zhang J, Fan M, Liang C, Cao J, Xu L. 2003. Lead exposure through gestation-only caused long-term learning/memory deficits in young adult offspring. Exp Neurol 184(1):489-95.

Yokoo EM, Valente JG, Grattan L, Schmidt SL, Platt I, Silbergeld EK. 2003. Low level methylmercury exposure affects neuropsychological function in adults. Environ Health 2(1):8.

Yoshida M, Watanabe C, Horie K, Satoh M, Sawada M, Shimada A. 2005. Neurobehavioral changes in metallothionein-null mice prenatally exposed to mercury vapor. Toxicol Lett 155(3):361-8.

Zhang HS, Song LH, Wang L, Qin YH. 2002. Lead can inhibit NMDA-, K(+)-, QA/KAinduced increases in intracellular free $\mathrm{Ca} 2+$ in cultured rat hippocampal neurons. Biomed Environ Sci 15(4):330-40. 


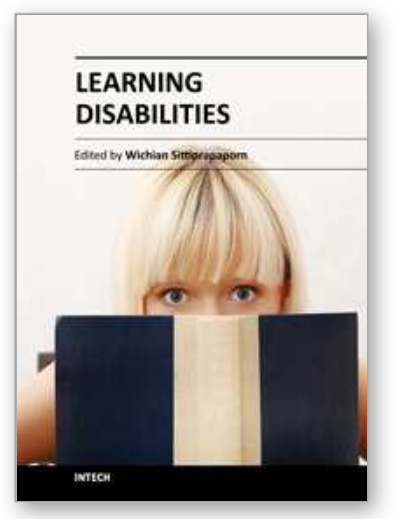

\author{
Learning Disabilities \\ Edited by Dr. Wichian Sittiprapaporn
}

ISBN 978-953-51-0269-4

Hard cover, 364 pages

Publisher InTech

Published online 14, March, 2012

Published in print edition March, 2012

Learning disability is a classification that includes several disorders in which a person has difficulty learning in a typical manner. Depending on the type and severity of the disability, interventions may be used to help the individual learn strategies that will foster future success. Some interventions can be quite simplistic, while others are intricate and complex. This book deserves a wide audience; it will be beneficial not only for teachers and parents struggling with attachment or behavior issues, but it will also benefit health care professionals and therapists working directly with special needs such as sensory integration dysfunction.

\title{
How to reference
}

In order to correctly reference this scholarly work, feel free to copy and paste the following:

Hideko Sone, Tin-Tin Win-Shwe, Xian-Yang Qin, Hiromi Akanuma and Satoshi Imanishi (2012). Environmental Chemical Substances in Relation to Neurodevelopmental Disorders: A Systematic Literature Review, Learning Disabilities, Dr. Wichian Sittiprapaporn (Ed.), ISBN: 978-953-51-0269-4, InTech, Available from:

http://www.intechopen.com/books/learning-disabilities/environmental-chemical-substances-in-relation-toneurodevelopmental-disorders-a-systematic-literatur

\section{INTECH}

open science | open minds

\section{InTech Europe}

University Campus STeP Ri Slavka Krautzeka 83/A 51000 Rijeka, Croatia Phone: +385 (51) 770447

Fax: +385 (51) 686166 www.intechopen.com

\section{InTech China}

Unit 405, Office Block, Hotel Equatorial Shanghai No.65, Yan An Road (West), Shanghai, 200040, China 中国上海市延安西路65号上海国际贵都大饭店办公楼405单元 Phone: +86-21-62489820

Fax: +86-21-62489821 
(C) 2012 The Author(s). Licensee IntechOpen. This is an open access article distributed under the terms of the Creative Commons Attribution 3.0 License, which permits unrestricted use, distribution, and reproduction in any medium, provided the original work is properly cited. 